\title{
Inducing cancer indolence by targeting mitochondrial Complex I is potentiated by blocking macrophage-mediated adaptive responses
}

\author{
Ivana Kurelac ${ }^{1,2}$, Luisa lommarini (10 ${ }^{3}$, Renaud Vatrinet ${ }^{1,3}$, Laura Benedetta Amato ${ }^{1}$, Monica De Luise ${ }^{1}$ \\ Giulia Leone ${ }^{3}$, Giulia Girolimetti ${ }^{1}$, Nikkitha Umesh Ganesh¹, Victoria Louise Bridgeman², Luigi Ombrato², \\ Marta Columbaro ${ }^{4}$, Moira Ragazzi ${ }^{5}$, Lara Gibellini ${ }^{6}$, Manuela Sollazzo ${ }^{3}$, Rene Gunther Feichtinger ${ }^{7}$, \\ Silvia Vidali (1D 7, Maurizio Baldassarre ${ }^{1}$, Sarah Foriel ${ }^{8,9}$, Michele Vidone ${ }^{1}$, Andrea Cossarizza6, \\ Daniela Grifoni id ${ }^{3}$, Barbara Kofler ${ }^{7}$, Ilaria Malanchi ${ }^{2}$, Anna Maria Porcelli ${ }^{3,10}$ \& Giuseppe Gasparre ${ }^{1,11}$
}

Converting carcinomas in benign oncocytomas has been suggested as a potential anti-cancer strategy. One of the oncocytoma hallmarks is the lack of respiratory complex I (Cl). Here we use genetic ablation of this enzyme to induce indolence in two cancer types, and show this is reversed by allowing the stabilization of Hypoxia Inducible Factor-1 alpha (HIF-1 $\alpha$ ). We further show that on the long run $\mathrm{Cl}$-deficient tumors re-adapt to their inability to respond to hypoxia, concordantly with the persistence of human oncocytomas. We demonstrate that $\mathrm{Cl}$-deficient tumors survive and carry out angiogenesis, despite their inability to stabilize HIF-1 $\alpha$. Such adaptive response is mediated by tumor associated macrophages, whose blockage improves the effect of $\mathrm{Cl}$ ablation. Additionally, the simultaneous pharmacological inhibition of $\mathrm{Cl}$ function through metformin and macrophage infiltration through PLX-3397 impairs tumor growth in vivo in a synergistic manner, setting the basis for an efficient combinatorial adjuvant therapy in clinical trials.

\footnotetext{
${ }^{1}$ Dipartimento di Scienze Mediche e Chirurgiche, Università di Bologna, Via Massarenti 9, 40138 Bologna, Italy. ${ }^{2}$ Tumor-Host Interaction Lab, The Francis Crick Institute, 1 Midland Rd, NW1 1AT London, UK. ${ }^{3}$ Dipartimento di Farmacia e Biotecnologie, Università di Bologna, Via Selmi 3 , 40126 Bologna, Italy. ${ }^{4}$ Laboratory of Musculoskeletal Cell Biology, IRCCS Istituto Ortopedico Rizzoli, Via Giulio Cesare Pupilli 1, 40136 Bologna, Italy. ${ }^{5}$ Anatomia Patologica, Azienda Ospedaliera S. Maria Nuova di Reggio Emilia, Viale Risorgimento 80, 42123 Reggio Emilia, Italy. ${ }^{6}$ Dipartimento di Scienze Mediche e Chirurgiche materno infantili e dell'adulto, Università degli Studi di Modena e Reggio Emilia, Via del Pozzo 71,41124 Modena, Italy. ${ }^{7}$ Research Program for Receptor Biochemistry and Tumor Metabolism, Department of Pediatrics, University Hospital of the Paracelsus Medical University, Muellner Hauptstraße 48, 5020 Salzburg, Austria. ${ }^{8}$ Khondrion BV, Philips van Leydenlaan 15, 6525 EX Nijmegen, The Netherlands. ${ }^{9}$ Radboud Center for Mitochondrial Medicine (RCMM) at the Department of Pediatrics, Radboud University Medical Center, Geert Grooteplein Zuid 10, 6500 HB Nijmegen, The Netherlands. ${ }^{10}$ Centro Interdipartimentale di Ricerca Industriale Scienze della Vita e Tecnologie per la Salute, Università di Bologna, Via Tolara di Sopra 41/E, 40064 Ozzano dell'Emilia, Italy. ${ }^{11}$ Centro di Ricerca Biomedica Applicata (CRBA), Università di Bologna, Via Massarenti 9, 40138 Bologna, Italy. These authors jointly supervised this work: Anna Maria Porcelli, Giuseppe Gasparre. Correspondence and requests for materials should be addressed to I.M. (email: ilaria.malanchi@crick.ac.uk) or to A.M.P. (email: annamaria.porcelli@unibo.it) or to G.G. (email: giuseppe.gasparre3@unibo.it)
} 
eveloping therapeutic strategies to target cancer metabolism is currently gaining momentum and one of the rising star metabolic approaches displaying antineoplastic potential involves inhibition of respiratory complex I $(\mathrm{CI})^{1-3}$, the first and rate-limiting enzyme of oxidative phosphorylation (OXPHOS). A profound revisiting of the seminal Warburg's hypothesis that tumors rely on aerobic glycolysis to fuel growth has led to establish a fundamental role for mitochondrial respiration in cancer progression. It is now accepted that highly aggressive, malignant cancer cells combine glycolytic and mitochondrial metabolic routes to meet energetic and biosynthetic demands ${ }^{4}$. Indeed, to maintain mitochondrial respiration, aggressive human cancers usually counterselect pathogenic mitochondrial DNA (mtDNA) CI mutations ${ }^{5-8}$. Conversely, severe mtDNA CI mutations are found in indolent, low-proliferative oncocytic tumors $^{8,9}$, i.e., neoplasms characterized by cells accumulating mostly dysfunctional, aberrant mitochondria and displaying scarce vasculature associated with destabilization of Hypoxia Inducible Factor-1 alpha (HIF-1 $\alpha$ ), the main promoter of vasculogenesis, glycolysis, and survival in hypoxic environment ${ }^{10}$. Oncocytomas represent an excellent case study in oncology, as they appear to be de facto short-circuited tumors that have become confined to a low-proliferative state due to metabolic constraints, likely deriving from the occurrence of high loads of pathogenic mtDNA mutations ${ }^{8,11}$ or from an impairment in autophagy ${ }^{12}$. Converting carcinomas into oncocytomas as an anti-cancer strategy has been proposed by targeting autophagy master regulator ATG $7^{13}$. Starting from the identification of genetic hallmarks of oncocytomas, i.e., severe mtDNA mutations in $\mathrm{CI}$, targeting this enzyme may be an even more efficient alternative approach to induce indolence, as this would simultaneously cause OXPHOS defects and the inability to adapt to hypoxia, shutting off several essential pathways in cancer cells. However, even if the severe CI damage could be expected to cause a metabolic catastrophe and impede malignant progression, oncocytic tumors linger in their indolent and slow-growing state, displaying quiescent but potentially perilous features of chemoresistance ${ }^{14,15}$. This is evident in human neoplasms rather than in mouse models, where reversion of the benign phenotype is technically difficult to assess for such slowgrowing cancers. It is therefore yet unclear how human CIdeficient tumors may promote angiogenesis despite HIF1 impairment. Thus, since modes of re-adaptation to CI dysfunction seem to exist, the identification of key factors keeping cancer cells alive is mandatory to design efficient combinatorial strategies to eradicate tumors. At the same time, to provide full justification for the use of CI inhibitors such as metformin in clinical practice, the dissection of the mechanisms linking CI inhibition to cancer growth arrest is warranted, especially those behind HIF-1a destabilization.

To fill the aforementioned gaps, we generated cancer cell lines lacking CI, via knockout of nuclear-encoded CI core subunit NDUFS3. Disengaging from the technical difficulties of dealing with mtDNA genetics, these models allow fine-tuning of NDUFS3 levels and subsequent CI activity.

We provide the proof of concept that $\mathrm{CI}$ ablation reduces tumorigenic potential and allows conversion into lowproliferative oncocytoma. Furthermore, while proving that the loss of HIF-1a is accountable for the decreased tumorigenic potential upon targeting CI, we additionally discover an atypical microenvironment response mediated by protumorigenic macrophages, which support survival of CI-deficient masses, and which we synergistically targeted to significantly increase therapeutic efficacy of metformin.

\section{Results}

NDUFS3 knockout induces a low-proliferative cancer phenotype. The molecular mechanisms linking CI impairment and reduction of tumorigenic potential have only been partially addressed, mainly due to difficult-to-handle cell models bearing mtDNA mutations. Thus, with the aim to demonstrate the antitumorigenic effect of CI deficiency and investigate the underlying mechanisms, we first generated easier-to-handle cancer cell models bearing a mtDNA-independent CI dysfunction. The nuclear-encoded NDUFS3 gene was knocked-out to induce CI deficiency in mesenchymal (osteosarcoma 143B) and epithelial (colorectal cancer HCT116) cancer cells, hereafter referred to as $143 \mathrm{~B}^{-/-}$and $\mathrm{HCT}^{-1-}$ (Supplementary Fig. 1), with the aim to generalize our findings in two cancer models of different tissue origin. Genetic ablation of NDUFS3 induced a severe decrease of CI NADH dehydrogenase activity (Supplementary Fig. 1d), reduced CI-driven ATP production (Supplementary Fig. 2a) and blocked mitochondrial respiration (Supplementary Fig. 2b), resulting in a major OXPHOS defect. On the other hand, NDUFS3 knockout induced an increase in glucose consumption (Supplementary Fig. 2c) and lactate production (Supplementary Fig. 2d), indicating that upregulation of glycolysis compensates the CI defect in vitro. Concordantly, the total amount of ATP was found to be comparable in CI-competent and deficient cells (Supplementary Fig. 2e). Moreover, the $\alpha$-ketoglutarate ( $\alpha-K G) /$ citrate ratio, as well as the $\mathrm{m}+5$ citrate enrichment after labeling with ${ }^{13} \mathrm{C}$-labeled glutamine, were markedly increased in CIdeficient cells, irrespective of the cellular background (Supplementary Fig. $2 \mathrm{f}-\mathrm{g}$ ). These data indicate that CI ablation triggers the preferential use of reductive carboxylation to supply the citrate pool, in agreement with previous studies in cancer cells with severe mtDNA mutations or treated with metformin ${ }^{16-18}$. Under selective pressures in vivo, the lack of NDUFS3 caused a significant decrease of xenograft growth in both cancer types (Fig. 1a) and reduced cell invasion (Fig. 1b), indicating that the metabolic reprogramming occurring following CI disruption (Supplementary Fig. 3a) allows cell survival but is not sufficient to fully recover the tumorigenic and invasive potential. $143 \mathrm{~B}^{-/-}$ and $\mathrm{HCT}^{-1-}$ tumors displayed lower KI-67 proliferation index (Fig. 1c) and no signs of necrosis, a known marker of aggressiveness, which was instead abundant in CI-competent tumors (Fig. 1d). The cytostatic effect of CI ablation was confirmed by a decreased caspase activation in the $\mathrm{KO}$ tumors, in agreement with what we previously reported ${ }^{19}$ (Supplementary Fig. 3b). Interestingly, CI-deficient masses were composed of eosinophilic cancer cells (Fig. 1d) harboring swollen mitochondria with deranged cristae (Fig. 1e, Supplementary Fig. 3c), well recapitulating oncocytic lesions, which was further strengthened by the finding that $143 \mathrm{~B}^{-1-}$ xenografts displayed an increased expression of PPARGC1A, the master regulator of mitochondrial biogenesis (Supplementary Fig. 3d). Re-expression of NDUFS3 in $143 \mathrm{~B}^{-1-}$ cells rescued CI activity (Supplementary Fig. 3e), mitochondrial ultrastructure and tumorigenic potential (Fig. 1f). Taken together, these data indicate that NDUFS3 knockout tumors recapitulated the bioenergetic features of lesions harboring mtDNA disruptive mutations, such as oncocytes, and provide the proof of principle for the anti-tumorigenic effect of CI damage.

Targeting CI converts malignant cancers in oncocytomas. After establishing that tumorigenic potential of CI-deficient tumors is reduced compared to their CI-competent counterpart, we sought to prove that depleting $\mathrm{CI}$ in a well-formed, progressing mass may represent an effective strategy to decrease or arrest growth in vivo. NDUFS3 was constitutively expressed in $143 \mathrm{~B}^{-/-}$cells 
a
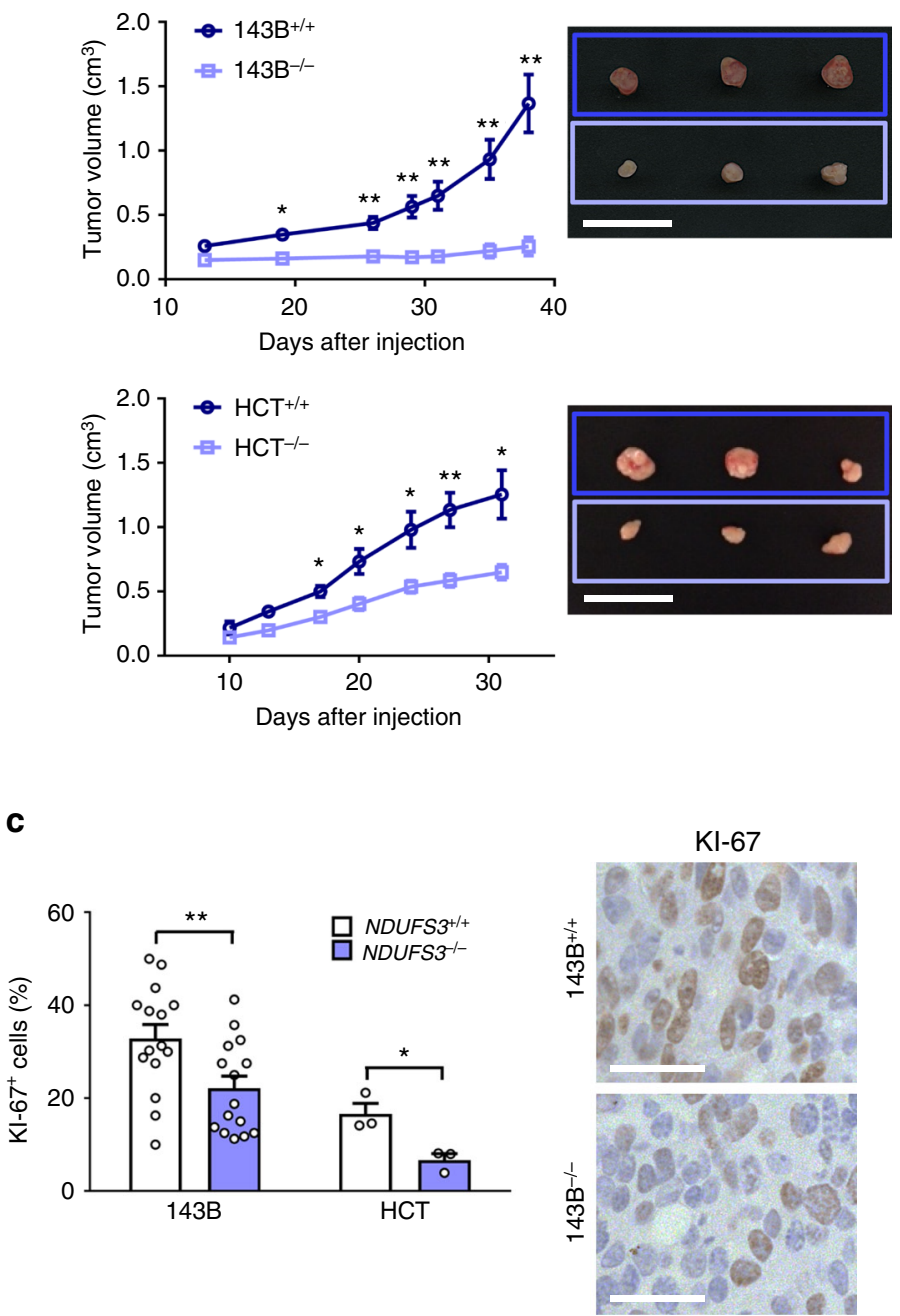

b

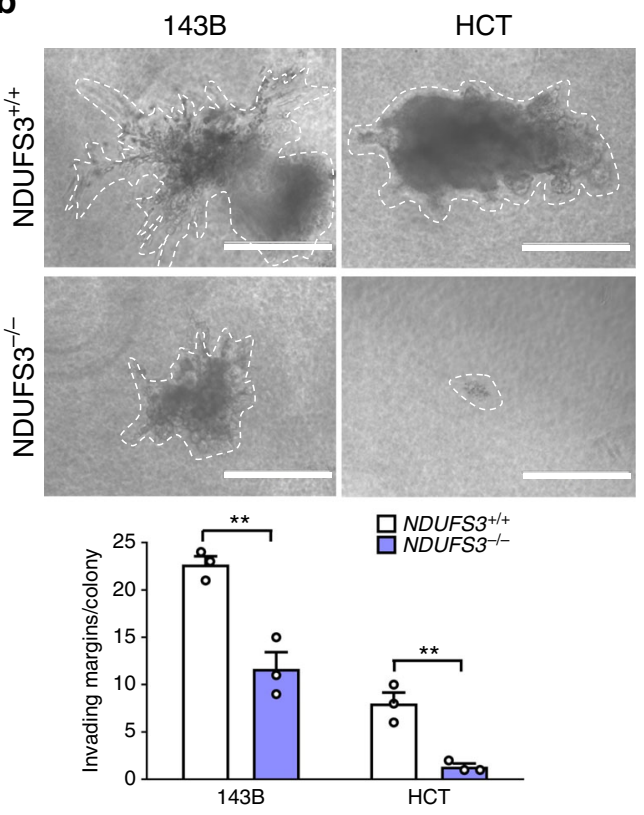

d

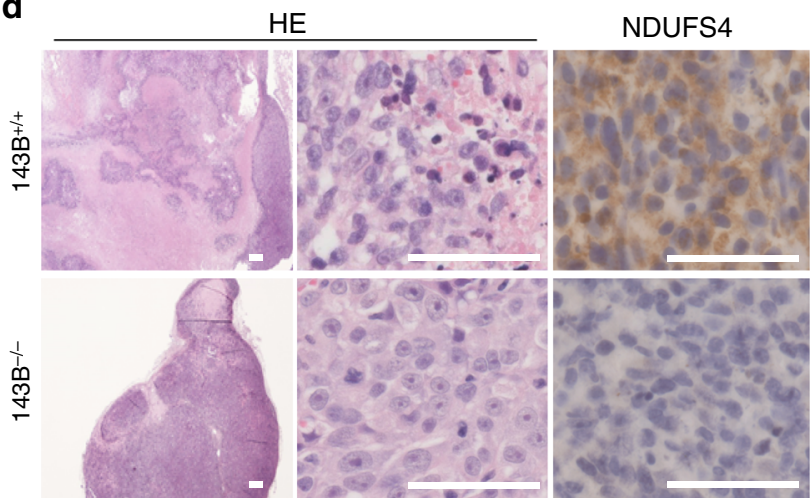

$\mathbf{f}$
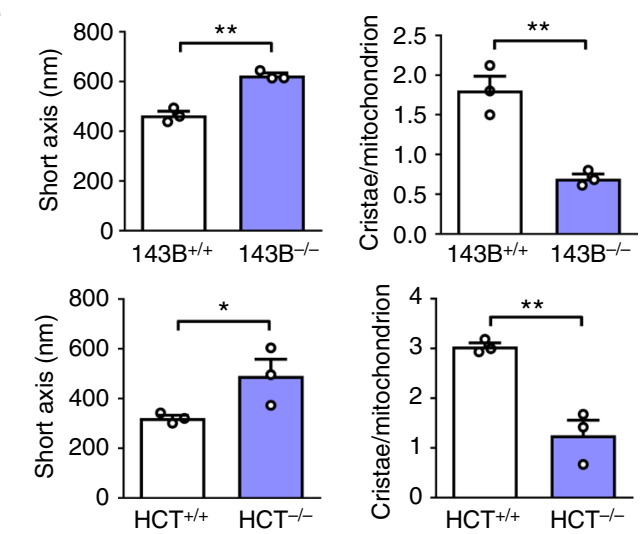
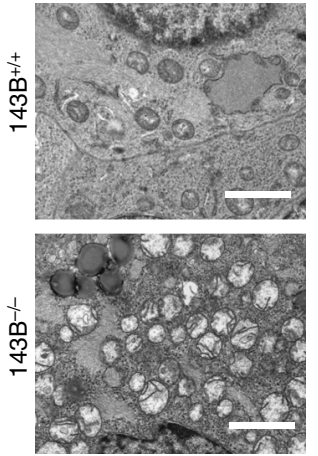

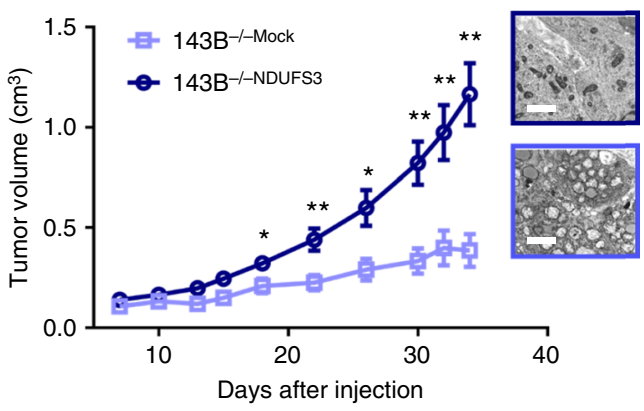

$\left(143 \mathrm{~B}^{-/-N D U F S} 3\right)$, under the control of a transactivator negatively regulated by doxycycline (Dox) (Supplementary Fig. 4a). By using Dox doses which did not affect CI assembly, a complete loss of the enzyme was observed after 9 days of treatment (Supplementary Fig. 4b). Moreover, at doses which do not impact tumor growth or mtDNA-encoded protein levels (Supplementary Fig. 4c-e), Dox-mediated induction of CI loss caused growth arrest in $85.7 \%$ of $143 \mathrm{~B}^{-/-N D U F S 3}$ proliferating tumors, decreased KI-67 proliferative index and resulted in significantly prolonged survival of the animals (Fig. 2a, b, Supplementary Fig. 5). CI-depleted tumors showed clear-cut negative
NDUFS3 staining (Fig. 2c), demonstrating complete loss of the protein and suggesting that no promotor leakage occurred. Staining for NDUFS4 subunit, a marker of CI assembly ${ }^{9}$, was reduced in Dox-treated $143 \mathrm{~B}^{-/-N D U F S 3}$ xenografts, indicating that the enzyme was successfully targeted (Fig. 2c). Furthermore, Dox-treated masses displayed ultrastructural mitochondrial alterations (Fig. 2d) and lack of necrosis at tumor leading margins (Fig. 2e), a phenotype resembling NDUFS3 knockout xenografts. All these data demonstrate that targeting CI during cancer progression converts malignant tumors into low-proliferative, oncocytoma-like tumors. 
Fig. 1 NDUFS3 knockout triggers oncocytic phenotype and reduces tumorigenic potential. a Growth curves of $143 B$ xenografts in CD-1 nude mice $[n=15$, $\mathrm{df}=28, t$ (day 38$)=5.4]$ and HCT xenografts in ICRF nude mice $[n=8, \mathrm{df}=14, t$ (day 31) $=3.6]$. Data are mean \pm s.e.m. Representative tumors are shown. Scale bars: $2 \mathrm{~cm}$. b Cell invasion assay in 3D collagen-l/Matrigel media of 143B and HCT xenograft-derived cells. Representative colonies are displayed and their margin delineated with dashed line. Scale bars: $400 \mu \mathrm{m}$. Quantification of invading areas of the colonies is shown $[n=3, \mathrm{df}=4, t(143 \mathrm{~B})=5.578$, $t(\mathrm{HCT})=5.547]$. Data are mean \pm s.e.m. c Quantification of cells displaying KI-67 positive nuclei in $143 \mathrm{~B}(n=15, \mathrm{df}=28, t=2.7)$ and $\mathrm{HCT}(n=3, \mathrm{df}=4$, $t=3.7)$ tumors. Representative image of KI-67 immunohistochemistry staining is shown. Scale bars: $25 \mu \mathrm{m}$. Data are mean + s.e.m. $\mathbf{d}$ Hematoxylin/eosin (HE) staining and immunohistochemistry for NDUFS3 and NDUFS4 Cl subunits. Representative images are shown. Scale bars: $50 \mu$ m. e Evaluation of short mitochondrial axis width and cristae number in 143B xenografts (axis: $n=3, \mathrm{df}=4, t=8.3$; cristae: $n=3$, df $=4, t=5.9$ ) and HCT cells (axis: data were log-transformed, $n=3, \mathrm{df}=4, t=2.8$; cristae: $n=3, \mathrm{df}=4, t=5.7)$. Representative electron micrographs are shown. Scale bars: $2 \mu \mathrm{m}$. Data are mean $+\mathrm{s}$. e.m. f Growth curves of 143B xenografts derived from cells carrying the empty vector (143B-/-Mock) or re-expressing wild-type NDUFS3 (143B-/-NDUFS3) in CD-1 nude mice. Data are mean \pm s.e.m. $[n=6, d f=10, t$ (day 34$)=5.2]$. Representative electron micrographs of the xenografts are shown. Scale bars: $2 \mu \mathrm{m}$. In each panel, statistical significance is specified with asterisks $\left({ }^{\star} p<0.05,{ }^{\star \star} p<0.01,{ }^{\star \star \star} p<0.001\right)$

Next, we corroborated our findings in a Drosophila melanogaster model of tumorigenesis, in which CI abolishment was feasible through the targeting of another crucial CI subunit, namely the ortholog of human NDUFV1. This allowed us to investigate whether the phenotype observed by targeting NDUFS3 might be extrapolated to any severe CI damage which would hamper specific $\mathrm{NADH}$ dehydrogenase activity, leading to NADH accumulation, as we previously showed ${ }^{19-21}$. A polaritydeficient strain was used, in which mutations of the evolutionarily conserved lethal giant larvae (lgl) tumor suppressor gene induce malignant growth of the epithelial larval organs which progressively grow into frank cancers, causing late individuals to die as pre-pupae with their anterior half completely filled by giant tumors $^{22}$. In this transformed context, we induced $G F P^{+} \lg l^{-1-}$ ndufv1 knockdown clones (hereafter referred to as $V 1^{\mathrm{KD}}$ ) at 6 days after egg laying (AEL), a time when neoplastic growth is obvious. As a control, $G F P^{+} l g l^{-1-l u c K D}$ clones were induced in the same experimental conditions. The epithelial tumors were isolated from $\mathrm{GFP}^{+}$larvae at 8 days AEL. The CI-deficient $V 1^{\mathrm{KD}}$ masses were smaller than controls (Fig. 2f, Supplementary Fig. 6a) and cell density analysis revealed an average of $50.75 \% \mathrm{GFP}^{+}$ cells in the $V 1^{\mathrm{KD}}$ samples versus $74.87 \%$ in the control counterparts (Fig. 2f). Subsequently, to investigate the phenotypic consequences of CI disruption in cells undergoing neoplastic transformation in an otherwise wild-type organ, mimicking mammalian cancer onset, we induced ndufv1 knockdown in a cooperative system, extensively used to recapitulate clonal carcinogenesis in Drosophila, where a $l g l$ loss of function mutation is combined with oncogenic Ras $\left(\operatorname{Ras}^{\mathrm{V} 12}\right)^{23}$. The $l g l^{-1-} \mathrm{Ras}^{\mathrm{V} 12}$ clones in which ndufv1 was knocked-down appeared considerably smaller than the control counterparts and showed a lower roundness coefficient (Supplementary Fig. $6 \mathrm{~b}-\mathrm{c})$, indicating poor capacity to form three-dimensional structures. The mitotic index measured by $\mathrm{PH} 3$ staining was reduced in $l g l^{-l-}$ Ras $^{\mathrm{V} 12} V 1^{\mathrm{KD}}$ tumors (Supplementary Fig. 6d), whereas cell death, defined by activation of caspase 3, was comparable between the $l g l^{-1-} \mathrm{Ras}^{\mathrm{V} 12} \mathrm{V1}^{\mathrm{KD}}$ and control samples (Supplementary Fig. 6e). These data confirmed that CI deficiency reduces tumorigenic potential by decreasing the proliferation rate of transformed cells, rather than inducing apoptosis, in line with our previous observations ${ }^{19}$. Altogether, the data collected in the two Drosophila cancer models sustained the findings obtained in the mammalian systems, confirming the essential role of CI in both initiation and progression of tumor growth, and indicating CI as a valid target for anti-cancer therapy.

HIF-1a loss is involved in CI-null tumor growth decrease. CI deficit in oncocytic tumors has been associated with lack of HIF-1a stabilization ${ }^{10}$, a phenomenon also observed in xenografts carrying severe mtDNA mutations ${ }^{19,21}$ or treated with metformin ${ }^{1}$. Indeed, HIF-1a stabilization was absent in the
$143 \mathrm{~B}^{-1-}$ and $\mathrm{HCT}^{-1-}$ tumors (Fig. 3a) and associated with a lower expression of HIF1-responsive genes (Fig. 3b). Moreover, $143 \mathrm{~B}^{-/-\mathrm{NDUFS} 3}$ xenografts, in which CI function was restored, showed the recovery of HIF-1 $\alpha$ activation, whereas Dox-induced CI loss caused HIF-1 $\alpha$ destabilization (Supplementary Fig. 7a). Finally, staining the Drosophila HIF-1a ortholog Similar showed lack of its nuclear accumulation in $l g l^{-/-} R a s^{\mathrm{V} 12} V 1^{\mathrm{KD}}$, which in the control clones was evident and followed by expression of invasion marker MMP1 (Fig. 3c), confirming HIF-1a destabilization as an evolutionarily conserved mechanism associated with CI deficiency. Interestingly, CI-deficient xenografts were prevalently negative for pimonidazole staining, used as the hypoxia marker, even when left to grow until the size comparable to that of CI-competent controls (10\% of the animal weight) (Supplementary Fig. 7b, c), suggesting that CI-deficient tumors barely ever acquire a hypoxic phenotype. The rare hypoxic foci identified in $143 \mathrm{~B}^{-1-}$ xenografts were negative for HIF-1a staining (Fig. 3d), allowing to hypothesize that CI deficiency causes HIF1a destabilization even in true hypoxia. To establish whether CI deficiency induces defects in HIF-1 $\alpha$ stabilization, we analyzed HIF-1a status under controlled hypoxic environment in vitro. $143 \mathrm{~B}^{-/-}$and $\mathrm{HCT}^{-/-}$cells grown under $1 \% \mathrm{O}_{2}$ conditions displayed a delayed HIF-1a stabilization (Fig. 3e) and lower expression of HIF1-responsive genes (Supplementary Fig. 7d), compared to their CI-competent counterparts, demonstrating an intrinsic defect in the HIF-1a stabilization pathway in CIdeficient tumors. With the aim to prove that the growth deficit of cells lacking functional CI was due to their inability to activate HIF1, a non-degradable and constitutively expressed form of HIF- $1 \alpha^{24}$, here named triple mutant (TM), was introduced into $143 \mathrm{~B}^{-1-}$ and $\mathrm{HCT}^{-/-}$cells. When compared to the empty vector controls $\left(143 \mathrm{~B}^{-/- \text {Mock }}\right.$ and $\left.\mathrm{HCT}^{-/- \text {Mock }}\right)$, cells constitutively expressing the TM-HIF-1a $\left(143 \mathrm{~B}^{-/-\mathrm{TM}}\right.$ and $\left.\mathrm{HCT}^{-/-\mathrm{TM}}\right)$ displayed HIF-1a stabilization, translocation into the nucleus and higher transcription of HIF1 downstream targets regardless of normoxia (Fig. 3f, Supplementary Fig. 8a, b), and formed larger colonies in 3D collagen/matrigel media (Supplementary Fig. 8c). Moreover, while TM-HIF-1a did not influence the tumorigenic potential of $143 \mathrm{~B}^{+/+}$cells (Supplementary Fig. $8 \mathrm{~d}$ ), CI-deficient cells expressing TM-HIF-1a formed larger xenografts when injected in nude mice (Fig. 3g, Supplementary Fig. 8e). Thus, the growth deficit of cells lacking functional CI was at least partially due to their inability to activate HIF1.

CI deficiency boosts PHD activity and destabilizes HIF-1 $\alpha$. We proceeded to explore the molecular mechanisms linking CI impairment with HIF-1a destabilization. The best-characterized mode of HIF-1a regulation is the PHDs-mediated hydroxylation, which in normoxia triggers its proteasomal degradation ${ }^{25}$. In addition, several PHD-independent mechanisms of HIF-1 $\alpha$ regulation have been identified ${ }^{26}$, such as reactive oxygen species 
a
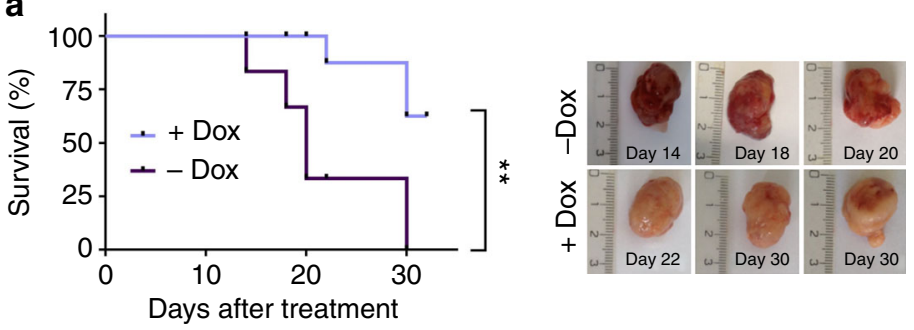

b
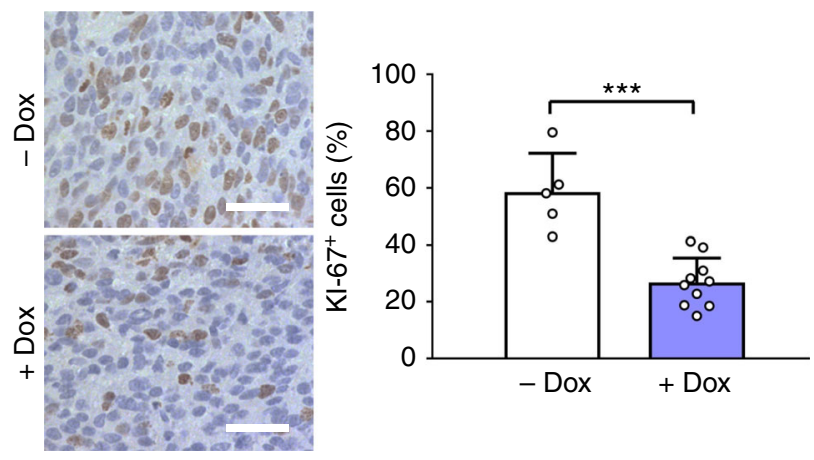

d
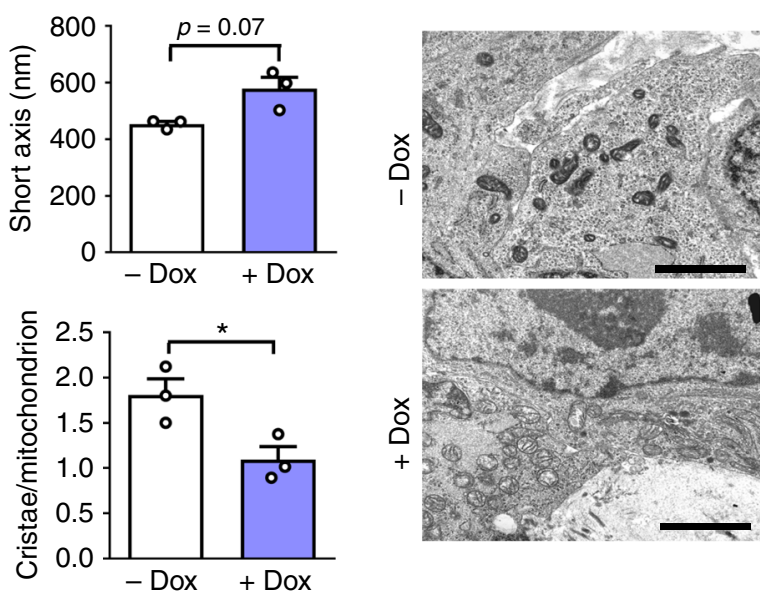

e
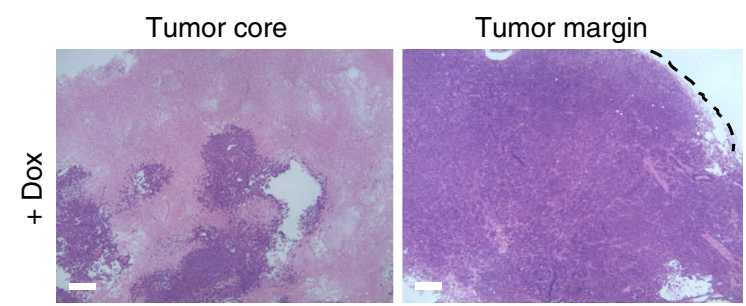

C
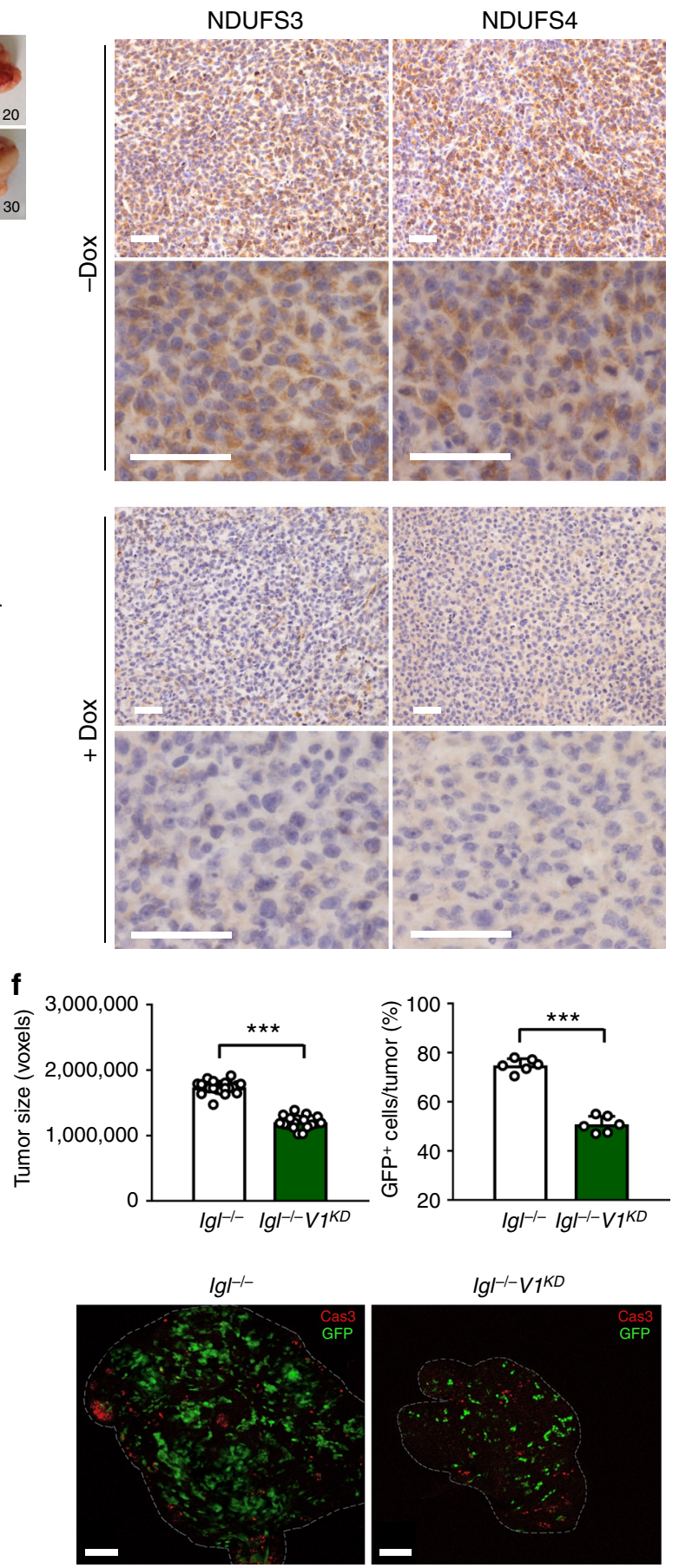

Fig. 2 Targeting $\mathrm{Cl}$ arrests tumor progression and converts carcinomas into oncocytomas. a Kaplan-Meier survival curves of CD-1 nude mice injected with $143 \mathrm{~B}^{-/- \text {NDUFS3 }}$ cells and treated with $(n=7)$ or without $(n=5)$ Dox $\left(1 \mathrm{mg} \mathrm{mL}^{-1}\right.$ in drinking water $)$. Survival end-point: xenografts reaching $10 \%$ of animal weight. Representative xenografts are shown. $\mathbf{b}$ Quantification of cells displaying KI-67 positive nuclei in 143B-/-NDUFS3 tumors treated with ( $n=7$ ) or without $(n=5)$ Dox ( $1 \mathrm{mg} \mathrm{mL}^{-1}$ in drinking water) ( $\left.\mathrm{df}=13, t=5.539\right)$. Representative image of KI-67 immunohistochemistry staining is shown. Scale bars: $50 \mu \mathrm{m}$. Data are mean + s.e.m. c Immunohistochemistry staining of $\mathrm{Cl}$ subunits in xenografts treated with or without Dox (1 $\mathrm{mg} \mathrm{mL}{ }^{-1}$ in drinking water). Representative images are shown. Scale bars: $50 \mu \mathrm{m}$. d Short mitochondrial axis width $(n=3, \mathrm{df}=4, t=2.4)$ and cristae number $(n=3, \mathrm{df}=4, t=3.1)$ evaluation, with representative electron micrographs of xenografts treated with or without Dox ( $1 \mathrm{mg} \mathrm{mL}^{-1}$ in drinking water). Scale bars: $2 \mu \mathrm{m}$. Data are mean + s.e.m. e Hematoxylin/eosin staining of $143 B^{-/-N D U F S 3}$ tumors treated with Dox. Tumor margin is indicated by the dashed line. Representative images are shown. Scale bars: $100 \mu \mathrm{m}$. $\mathbf{f}$ Immunofluorescence for caspase 3 (Cas3) of $\mathrm{Cl}$-competent $\left(|\mathrm{g}|^{-/-}\right.$) and deficient $\left(\left.\mathrm{Ig}\right|^{-/-} \mathrm{V} 7^{K D}\right.$ ) epithelial tumors $\left(\mathrm{GFP}^{+}\right)$of the fly. Dashed lines delineate tumor margin. Scale bars: $80 \mu \mathrm{m}$. Tumor size $(n=25, \mathrm{df}=48, t=13.2)$ and the average number of GFP+ cells $(n=6, \mathrm{df}=10, t=13.8)$ were evaluated. Data are mean + s.e.m. In each panel, statistical significance is specified with asterisks $\left({ }^{\star} p<0.05,{ }^{\star \star} p<0.01\right.$, $\left.{ }^{\star \star *} p<0.001\right)$ 

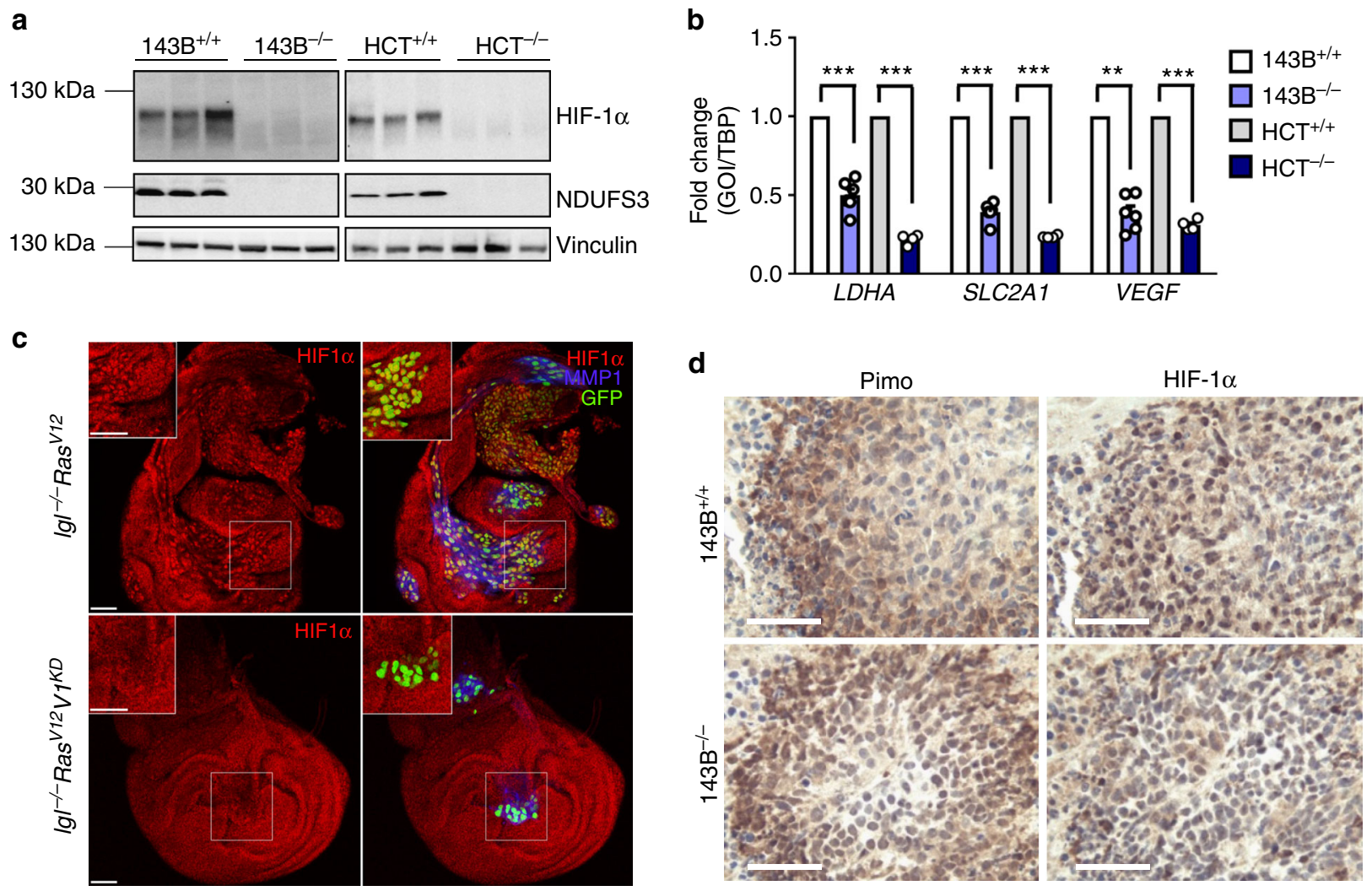

e
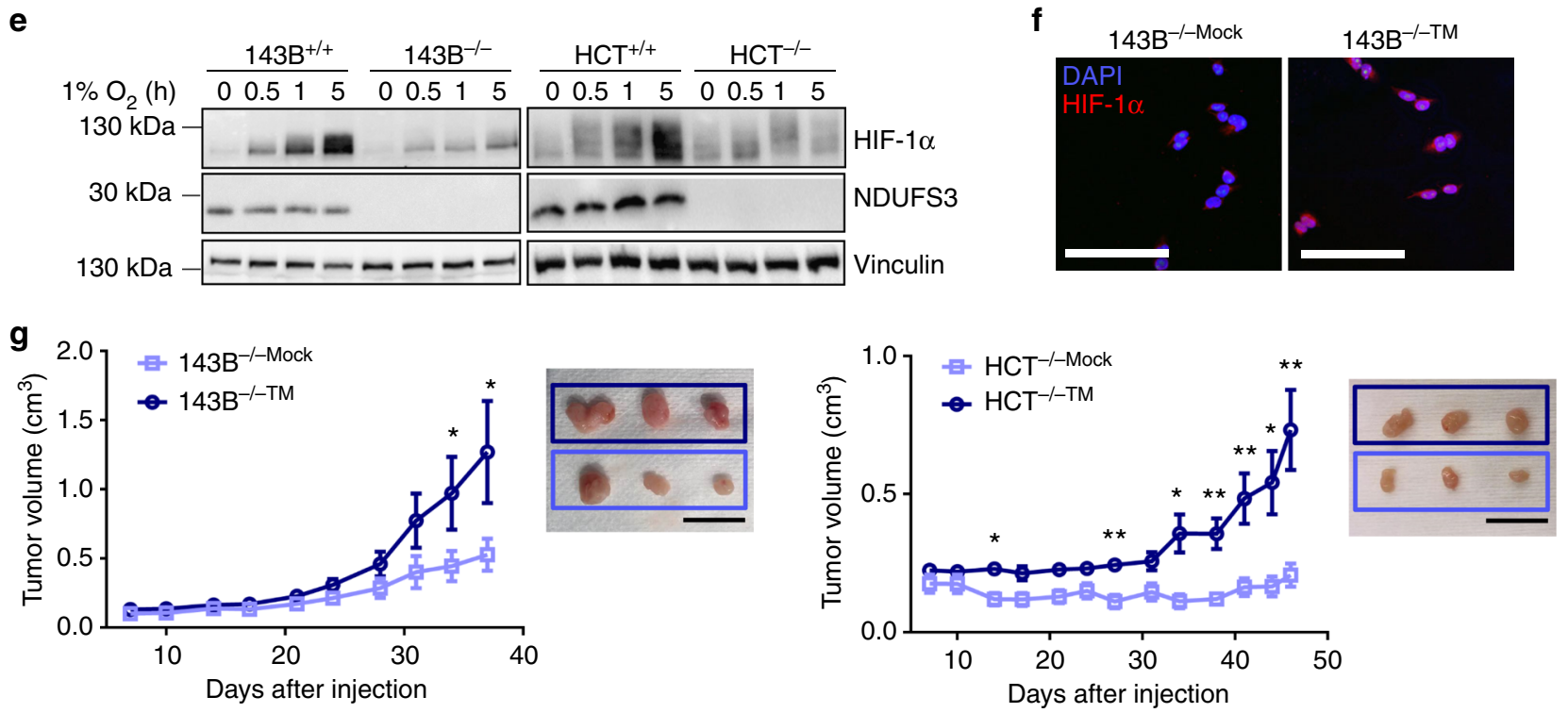

Fig. $3 \mathrm{Cl}$ deficiency promotes HIF-1 $\alpha$ destabilization. a HIF-1 $\alpha$ and NDUFS3 western blot analysis in $143 \mathrm{~B}$ and $\mathrm{HCT}$ xenografts. Vinculin was used as loading control. b Gene expression of HIF-1 $\alpha$ targets evaluated by qRT-PCR in 143B $[n=8, \mathrm{df}=14, t(L D H A)=6.5, t(S L C 2 A 1)=4.5, t(V E G F)=4.7]$ and HCT $[n=4, \mathrm{df}(L D H A)=6, t(L D H A)=14.1, \mathrm{df}(S L C 2 A 1)=3, t(S L C 2 A 1)=9.3, \mathrm{df}(V E G F)=6, t(V E G F)=11.7)]$ xenografts. For SLC2A1 in HCT cells the Student's $t$-test assuming unequal variance was applied. Data are mean + s.e.m. c Immunofluorescence for HIF-1 $\alpha$ and MMP1 in Drosophila wing discs. Tumor tissue is composed of GFP ${ }^{+}$cells. Representative images are shown. Scale bars: $40 \mu \mathrm{m}$. $\mathbf{d}$ Immunohistochemical staining of pimonidazole (Pimo)-labeled protein adducts and HIF- $1 \alpha$ in $143 \mathrm{~B}^{+/+}$and $143 \mathrm{~B}^{-/-}$tumors of equivalent volume $\left(2 \mathrm{~cm}^{3}\right)$. Representative images are shown. Scale bars: $50 \mu \mathrm{m}$. e HIF- $1 \alpha$ and NDUFS3 western blot of $143 \mathrm{~B}$ and $\mathrm{HCT}$ cells cultured in $1 \% \mathrm{O}_{2}$ at indicated times. Vinculin was used as loading control. $\mathbf{f}$ Immunofluorescent staining of nuclei (DAPI) and HIF-1 $\alpha$ in $143 \mathrm{~B}^{-/-}$cells carrying empty vector (Mock) or TM-HIF-1 $\alpha$ (TM). Representative images are shown. Scale bars: $100 \mu$ m. $\mathbf{g}$ Growth curves in CD-1 nude mice of $143 \mathrm{~B}[n=15, \mathrm{df}=28, t($ day 37$)=2.2]$ and HCT [data were log-transformed, $n=7, \mathrm{df}=12, t($ day 46$)=3.5$ ] xenografts derived from $\mathrm{Cl}$-deficient cells carrying empty vector (Mock) or TM-HIF-1 $\alpha$ (TM). Data are mean \pm s.e.m. Representative tumors are shown. Scale bars: $2 \mathrm{~cm}$. In each panel, statistical significance is specified with asterisks $\left({ }^{\star} p<0.05,{ }^{\star \star} p<0.01,{ }^{\star \star \star} p<0.001\right)$ 
(ROS)-mediated activation of PI3K/Akt or ERK pathways which promote HIF1A transcription and translation ${ }^{27}$. No significant differences in $\mathrm{H}_{2} \mathrm{O}_{2}$ production and, concordantly, in the total glutathione level, between CI-competent and deficient cells were observed (Supplementary Fig. 9a, b), and the latter displayed no decrease but rather an increase in HIF1A mRNA levels compared to their control counterparts (Fig. 4a), indicating that HIF-1 $\alpha$ destabilization in CI-deficient cells does not involve mechanisms affecting HIF1A transcription. Furthermore, HIF-1a recovery was observed after treatment with the proteasome inhibitor MG132 (Fig. 4b), indicating that the lack of CI does not affect HIF-1 $\alpha$ translation. In particular, MG132 caused higher accumulation of hydroxylated HIF-1a (HIF-1a-OH) in $143 \mathrm{~B}^{-1-}$ and $\mathrm{HCT}^{-1-}$ cells (Fig. 4b, c), indicating an enhanced PHD activity in CIdeficient models. Recovery of HIF-1a protein was also observed in $143 \mathrm{~B}^{-1-}$ and $\mathrm{HCT}^{-1-}$ cells after treatment with dimethyloxallyl glycine (DMOG), a selective PHDs inhibitor ${ }^{28}$ (Fig. 4b), confirming CI deficiency determines a deregulation in the PHDmediated degradation of the protein. Of note, PHD1/2 protein levels were comparable between CI-competent and deficient cells (Supplementary Fig. 9c). In agreement with our previous data showing NADH-mediated $\alpha-K G$ accumulation in tumors carrying CI-disruptive mtDNA mutations ${ }^{19,21}$, CI-deficient cells displayed a significant increase in $\alpha-K G$ levels compared to controls, as well as in malate/aspartate ratio (Fig. $4 \mathrm{~d}$ ), the latter being an indication of NADH accumulation. $\alpha-\mathrm{KG}$ is known to boost PHD a

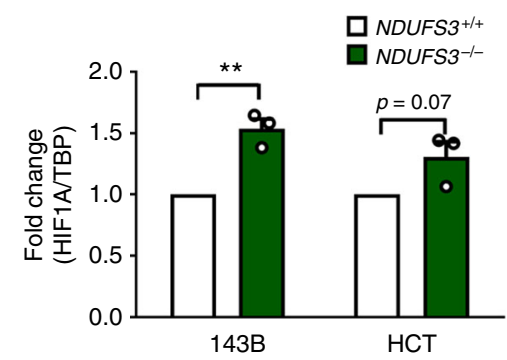

b

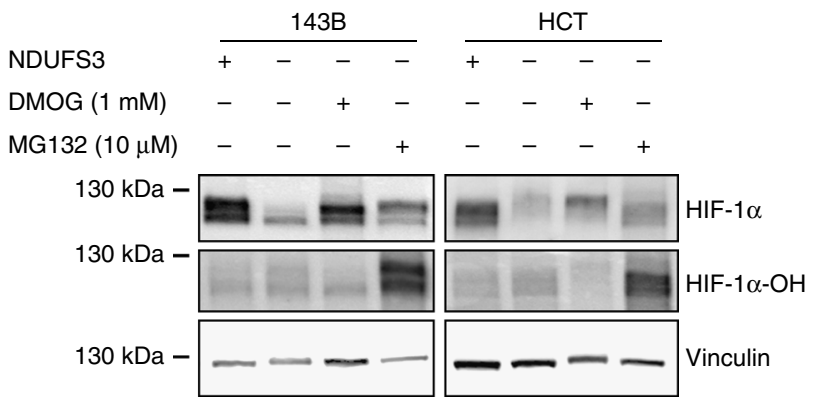

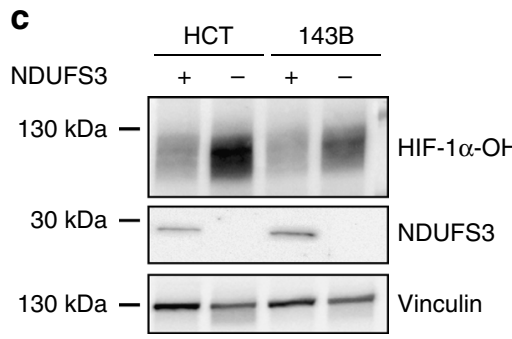

d
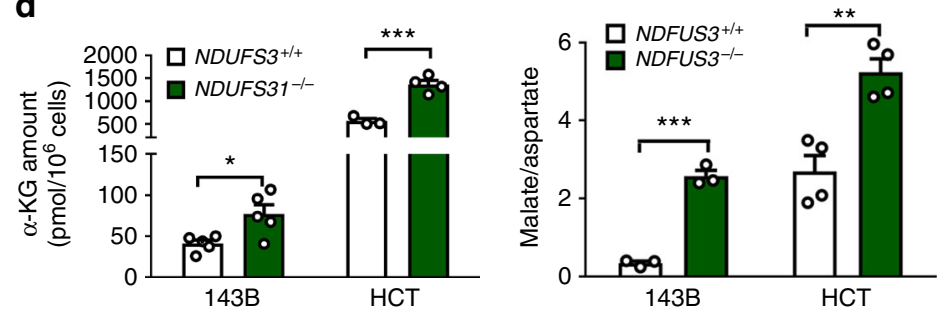

e

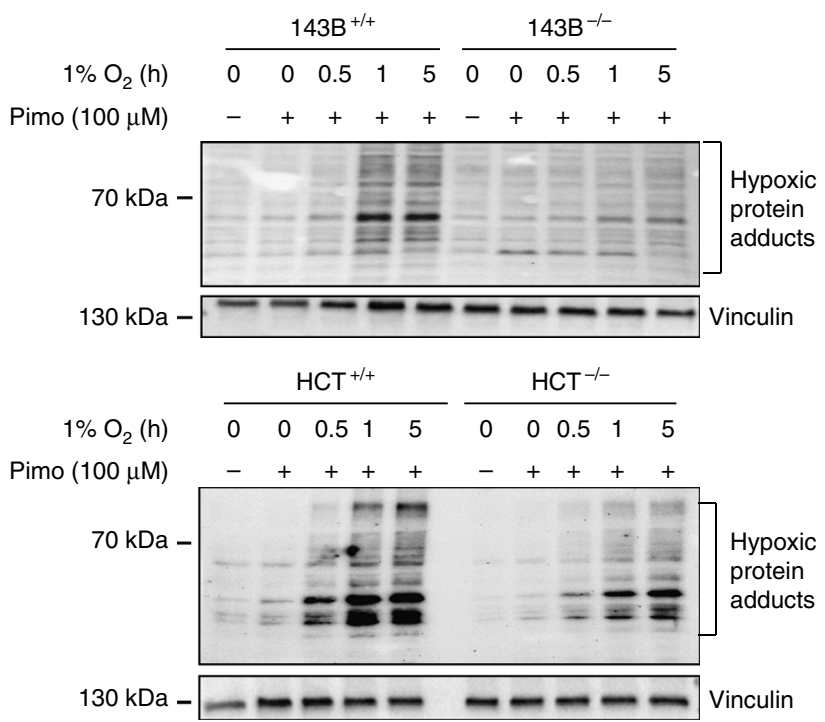

$\mathbf{f}$

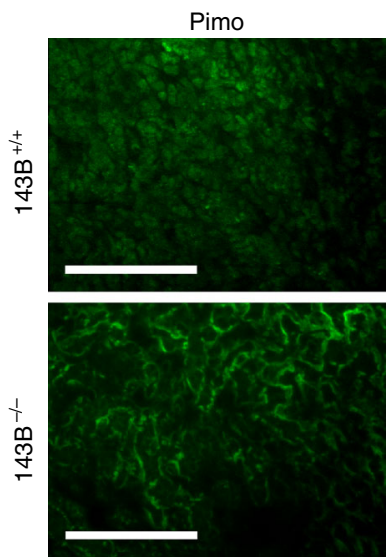

Fig. $4 \mathrm{Cl}$ deficiency induces HIF-1 $\alpha$ destabilization via increased PHD activity. a HIF1A expression level evaluated by $\mathrm{qRT}-\mathrm{PCR}$ in $143 \mathrm{~B}(n=3, \mathrm{df}=4, t=5.2)$ and $\mathrm{HCT}(n=3, \mathrm{df}=4, t=3.1)$ cells cultured for $5 \mathrm{~h}$ in $1 \% \mathrm{O}_{2}$. Data are mean + s.e.m. b HIF- $1 \alpha$ and HIF- $1 \alpha-\mathrm{OH}$ western blot analysis in $143 \mathrm{~B}$ and HCT cells exposed to hypoxia ( $1 \% \mathrm{O}_{2}$ for $5 \mathrm{~h}$ ) and treated with DMOG ( $1 \mathrm{mM}$ for $3 \mathrm{~h}$ ) or MG132 (10 $\mu \mathrm{M}$ for $3 \mathrm{~h}$ ). Vinculin was used as loading control. c HIF- $1 \alpha-\mathrm{OH}$ and NDUFS3 western blot analysis in 143B and HCT cells exposed to hypoxia ( $1 \% \mathrm{O}_{2}$ for $5 \mathrm{~h}$ ) and treated with MG132 (10 $\mu \mathrm{M}$ for $3 \mathrm{~h}$ ). Vinculin was used as loading control. $\mathbf{d} \alpha-K G$ amount and malate/aspartate ratio in $143 \mathrm{~B}[n(\alpha-\mathrm{KG})=2 ; n$ (malate/aspartate $)=3$, df $($ malate/aspartate $)=4, t($ malate $/$ aspartate $)$ $=14.3]$ and $\mathrm{HCT}[n=4, \mathrm{df}=6, t(\alpha-\mathrm{KG})=6.8, t$ (malate/aspartate $)=4.8]$ cells measured under basal conditions. Data are mean $+\mathrm{s}$.e.m. e Protein adducts western blot analysis in cells incubated with pimonidazole (Pimo) (100 $\mu \mathrm{M}$ for $1 \mathrm{~h}$ ). Vinculin was used as loading control. f Representative images of pimonidazole (Pimo) immunofluorescent staining in 143B xenografts. Scale bars: $100 \mu \mathrm{m}$. In each panel, statistical significance is specified with asterisks $\left({ }^{\star \star} p<0.01,{ }^{\star \star \star} p<0.001\right)$ 
activity by increasing its affinity for oxygen ${ }^{29,30}$. Furthermore, it has been shown that severe mitochondrial damage may cause a sufficient reduction in oxygen consumption to increase cytosolic oxygen concentrations regardless of the external hypoxia ${ }^{31}$. Thus, we evaluated the intracellular oxygen status of CI-deficient cells with the hypoxia marker pimonidazole and observed that, when grown in controlled in vitro hypoxic conditions, $143 \mathrm{~B}^{-/-}$and $\mathrm{HCT}^{-1-}$ cells experienced less intrinsic hypoxia than their CIcompetent counterparts (Fig. 4e), in accordance with the mainly normoxic condition observed in CI-deficient xenografts (Fig. $4 \mathrm{f}$ and Supplementary Fig. 7c). Taken together, these data imply that the delay and reduction of HIF-1a stabilization in CI-deficient cancer cells was caused by simultaneous increase in $\alpha-K G$ concentrations and reduced oxygen consumption, which together lead to a boost of PHD activity regardless of external hypoxia.

CI-deficient tumors display stroma-associated angiogenesis. Despite the lack of HIF-1 $\alpha$ and lower tumorigenic potential, CIdeficient masses, like oncocytomas, continued to progress (Supplementary Fig. 7b), meaning they probably engage alternative, HIF-1 $\alpha$-independent mechanisms to ensure nutrient supply. In the context of proposing CI targeting as an anti-cancer strategy, such growth persistence might present a potential risk of malignancy. Thus, we next investigated the consequences of HIF-1 $\alpha$ destabilization on angiogenesis in CI-deficient tumors, with the aim to identify the pro-survival adaptive mechanisms activated during their development. By staining with endothelial markers endomucin and CD31, we compared the vessels morphology in CI-competent and deficient masses. CI-competent tumors presented with vessels characterized by a large lumen and positivity for the pericyte marker smooth muscle actin (SMA), indicating mature and well-perfused vasculature (Fig. 5a). Moreover, these vessels were located in areas surrounded by hypoxia and HIF-1apositive cancer cells (Supplementary Fig. 10a), suggesting that they were most likely generated via HIF1-mediated signals. On the other hand, CI-deficient tumor vasculature in both $143 \mathrm{~B}^{-1-}$ and $\mathrm{HCT}^{-1-}$ xenografts was characterized mainly by small, lumen-free and SMA-negative vessels (Fig. 5a), indicating an impairment in vascular maturation. The re-expression of NDUFS3 in $143 \mathrm{~B}^{-/-}$cells restored HIF-1 $\alpha$-associated vasculature (Supplementary Fig. 10b), whereas tumors in which CI was depleted by Dox treatment displayed a greater number of SMAnegative vessels than their untreated controls (Supplementary Fig. 10c), demonstrating that immature vasculature was a consequence of CI dysfunction. Moreover, complementing $143 \mathrm{~B}^{-/-}$ and $\mathrm{HCT}^{-1-}$ cells with TM-HIF-1 $\alpha$ also rescued vessel maturation (Supplementary Fig. 10d), indicating that lack of an efficient vasculogenesis contributed to the lower tumorigenic potential of CI-deficient tumors. Since SMA is also a marker of cancer associated fibroblasts (CAFs), its staining showed that mature lumenbearing vessels in $143 \mathrm{~B}^{-1-}$ and $\mathrm{HCT}^{-1-}$ xenografts were located almost exclusively in the murine stromal component of the tumor (Fig. 5a), an observation corroborated by staining collagen fibers (Fig. 5b). Furthermore, as we previously demonstrated, xenografts derived from cell lines carrying high loads $(>85 \%)$ of CI disruptive m.3571insC/MT-ND1 mtDNA mutation ${ }^{19}$ also showed more pericyte-negative endothelial structures than their CIcompetent controls and displayed lumen-bearing vessels mainly in the stromal component (Supplementary Fig. 10e). Overall, histology revealed that the stromal component is more prominent in CI-deficient than in CI-competent tumors, where it was located mainly on the tumor periphery (Fig. $5 \mathrm{c}$ ). We next quantified the contribution of non-cancer cells in CI-deficient tumors at day 10 and day 30 post injection, to reconstruct the timeline of their recruitment. At day 30, corroborating the histology results, both
143B and HCT xenografts displayed higher stromal contribution in association with CI deficiency (Fig. 5d). These data, collected from both epithelial and mesenchymal tumors, and from different models of CI dysfunction, identified a more general rearrangement of tumor microenvironment in CI-deficient tumors, possibly involved in supporting their proliferation. In particular, since the stromal component of CI-deficient tumors was associated with their vasculature, we hypothesize that the microenvironment may be involved in compensatory mechanisms triggered to overcome the inability of CI-deficient tumors to activate HIF-1 $\alpha$-mediated angiogenesis.

Macrophage infiltration is a hallmark of CI-deficient tumors. The role of microenvironment in supporting tumor growth is well acknowledged, in particular as a source of pro-angiogenic factors $^{32}$. However, the microenvironment component in tumors may be highly heterogeneous and exert both protumorigenic and anti-tumorigenic functions. Thus, to identify the components which might support CI-deficient tumor growth, we next characterized the contribution of immune cells $(\mathrm{CD} 45+)$ and fibroblasts (CD31-CD45-) (Supplementary Fig. 11a). At day 10, no difference was observed in 143B xenografts, whereas $\mathrm{HCT}^{-1-}$ tumors showed an increased contribution of each of the populations analyzed (Supplementary Fig. 11b). At day 30, both 143B and HCT CI-deficient xenografts harbored more CD45 + cells than their CI-competent counterparts (Supplementary Fig. 11b), identifying immune cells abundance as a phenomenon shared in the CI-deficient tumor microenvironment. Thus, we further characterized the populations of the innate immune system in our models, namely macrophages (F4/80 + Lys6G-), neutrophils (Lys6G + F4/80-), natural killer cells $(\mathrm{NK}, \mathrm{CD} 49 \mathrm{~b}+$ ), and dendritic cells (CD11c + F4/80-) (Supplementary Fig. 11c). Of note, a higher number of necrosis-associated neutrophils was observed in CI-competent tumors (Fig. 6a and Supplementary Fig. 11d). The most striking and consistent difference we observed involved a higher number of macrophages in both 143B and HCT CIdeficient xenografts (Fig. 6a), particularly evident from the increase in the macrophage-to-cancer cells ratio (Fig. 6b). With the aim to investigate whether the higher macrophage abundance may be due to increased monocyte differentiation, we analyzed CD11b and Ly6C markers at day 30 (Supplementary Fig. 12a). Both tumor types harbored comparable numbers of classical CD11b + Ly6C + monocytes, whereas the number of differentiated CD11b + LyC- monocytes was higher in CIdeficient masses (Supplementary Fig. 12b). Indeed, the number of $\mathrm{CD} 11 \mathrm{~b}+\mathrm{Ly} 6 \mathrm{C}-\mathrm{F} 4 / 80+$ cells was also higher in CI-deficient tumors (Fig. 6c), suggesting that $\mathrm{CI}$ deficiency in cancer may promote differentiation of $\mathrm{CD} 11 \mathrm{~b}+\mathrm{Ly} 6 \mathrm{C}+$ cells into macrophages $(\mathrm{CD} 11 \mathrm{~b}+\mathrm{Ly} 6 \mathrm{C}-\mathrm{F} 480+)$. Strikingly, histology both at day 10 and at day 30 (Fig. 6c), revealed a difference in macrophage localization. While in CI-competent tumors they were restricted mainly at the tumor periphery, in CI-deficient tumors macrophages were found infiltrating the cancer mass (Fig. 6d). In line with this observation, we identified the downregulation of macrophage migration inhibitory factor (MIF) in CIdeficient cancer cells (Supplementary Fig. 13a). Interestingly, MIF is a HIF1-induced protumorigenic cytokine whose downregulation has recently been associated with macrophagemediated angiogenesis ${ }^{33}$. Indeed, we corroborated reduced levels of MIF in $143 \mathrm{~B}^{-1-}$ tumors and established that the complementation with TM-HIF-1a rescues MIF expression and reduces the number of macrophages in $143 \mathrm{~B}^{-1-}$ tumors (Supplementary Fig. 13b-d), suggesting the increased presence of macrophages in CI-deficient tumors may be a consequence of HIF1 inactivation. 
a
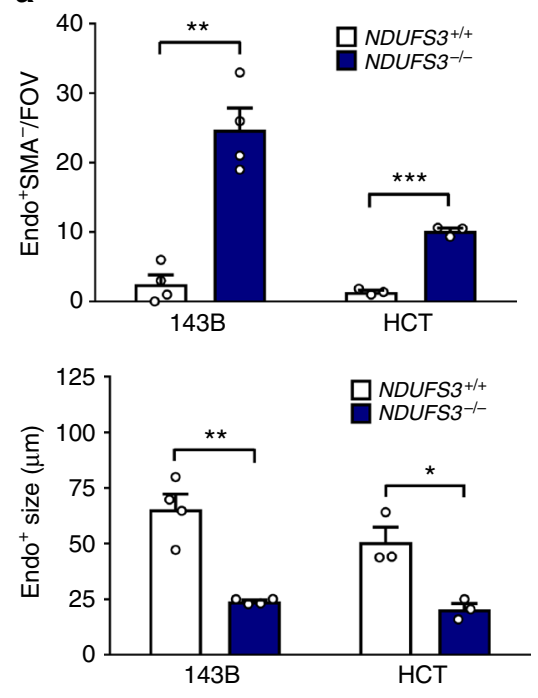

b

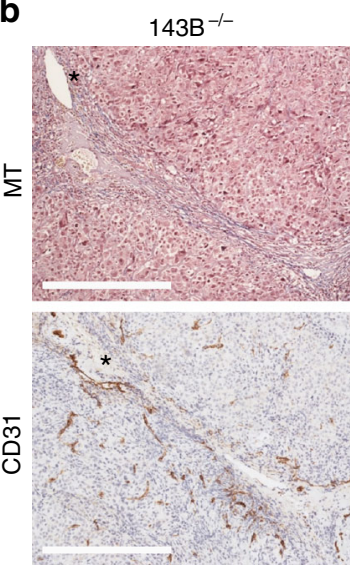

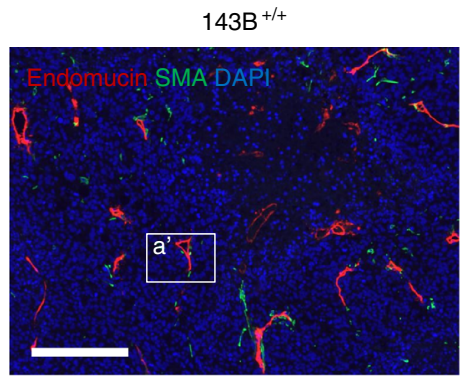

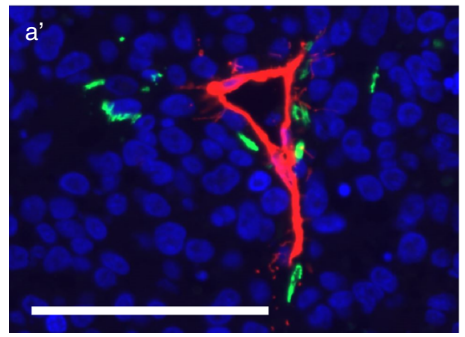

C
SMA
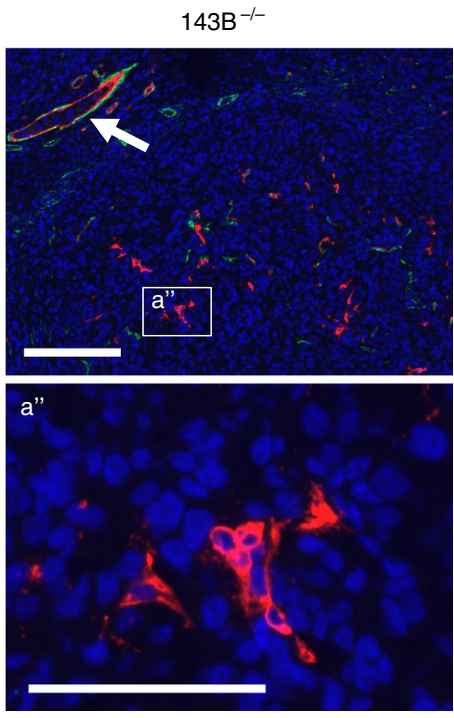

MT
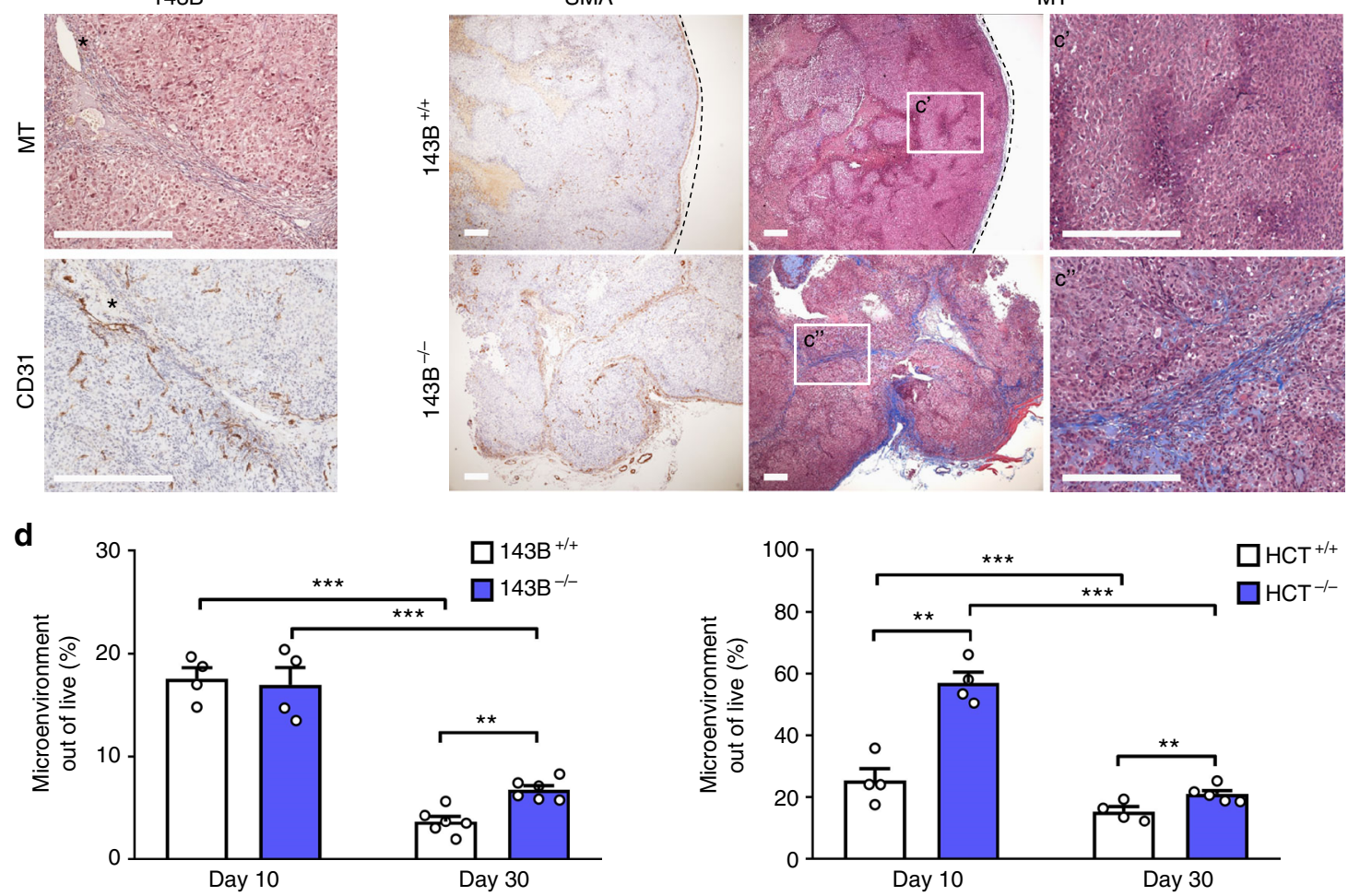

Fig. $5 \mathrm{Cl}$-deficient tumors display stroma-associated angiogenesis. a Number of pericyte-negative (Endo ${ }^{+} \mathrm{SMA}^{-}$) vessels per field of view (FOV) and average size of Endo ${ }^{+}$formations in $143 \mathrm{~B}\left[n=4, \mathrm{df}\left(\right.\right.$ Endo $\left.^{+} \mathrm{SMA}^{-}\right)=6, t\left(\right.$ Endo $\left.\left.^{+} \mathrm{SMA}^{-}\right)=6.6, \mathrm{df}(\mathrm{size})=3, t(\operatorname{size})=6\right]$ and $\mathrm{HCT}[n=3, \mathrm{df}=4, t$ $\left(E_{n d o}{ }^{S M A}{ }^{-}\right)=18.5, t($ size $\left.)=4.2\right]$ xenografts. For vessel size in $143 \mathrm{~B}$ xenografts, the Student's $t$-test assuming unequal variance was applied.

Representative images of immunofluorescent staining analyzing vessel morphology are shown. The arrow indicates a lumen-bearing vessel in the stromal area of a Cl-deficient tumor. Scale bars: $100 \mu \mathrm{m}$. Data are mean + s.e.m. b Masson's trichrome (MT) and CD31 immunohistochemistry of Cl-deficient xenografts. Representative images are shown. Scale bars: $100 \mu \mathrm{m}$. c Masson's trichrome (MT) and smooth muscle actin (SMA) staining in 143B $+/+$ and $143 \mathrm{~B}^{-/-}$xenografts. Dashed lines delineate the tumor margin. Scale bars: $100 \mu \mathrm{m}$. d Flow cytometry analysis of murine (stromal) cells in $143 \mathrm{~B}$ and $\mathrm{HCT}$ xenografts at day $10[n=4, \mathrm{df}=6, t(143 \mathrm{~B})=0.3, t(\mathrm{HCT})=6.2]$ and day $30[n(143 \mathrm{~B})=6, \mathrm{df}(143 \mathrm{~B})=10, t(143 \mathrm{~B})=4.8, n(\mathrm{HCT})=4, \mathrm{df}(\mathrm{HCT})=6$, $t(\mathrm{HCT})=2.9]$ post injection. Data are mean + s.e.m. In each panel, statistical significance is specified with asterisks $\left({ }^{\star} p<0.05,{ }^{\star \star} p<0.01,{ }^{\star \star \star} p<0.001\right)$

Since changes in the CAFs distribution were observed on histology (Fig. $5 \mathrm{c}$ and Fig. 6e), and since both CAFs and tumor associated macrophages (TAMs) have been shown to exert wellcharacterized pro-angiogenic roles ${ }^{32,34}$, it is reasonable to hypothesize they might converge in promoting survival in CI-deficient tumors. However, in line with the flow cytometry data at day 30 , the number of single CAFs in the tumor was comparable between the two groups (Supplementary Fig. 14a), indicating that CI-competent and deficient cells were equally able to activate fibroblasts, which was also confirmed in vitro (Supplementary Fig. 14b). On the other hand, the number of single macrophages was higher in CI-deficient tumors than in control masses (Fig. 6d), suggesting that 
a
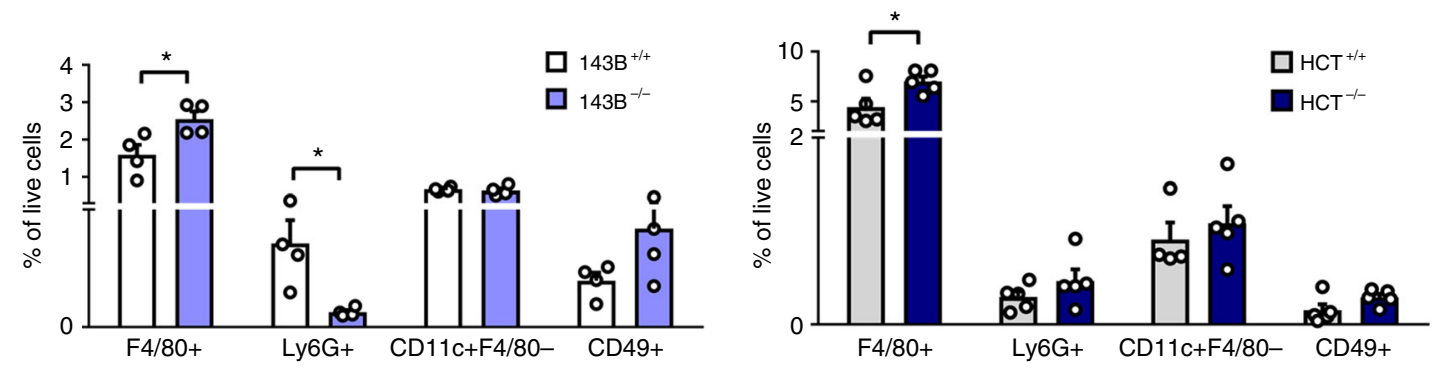

b
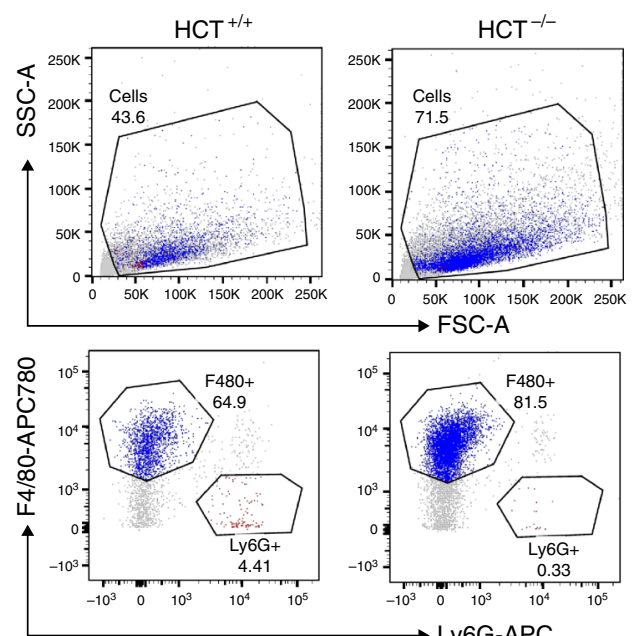

Gated on CD45+CD11b+

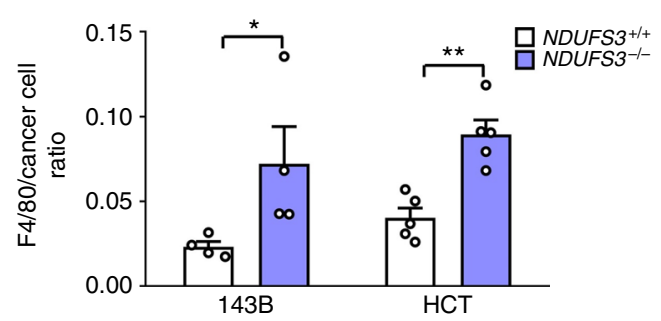

C

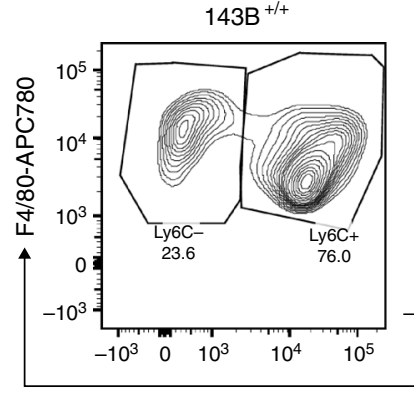

Gated on $\mathrm{CD} 45+\mathrm{CD} 11 \mathrm{~b}+\mathrm{F} 4 / 80+$

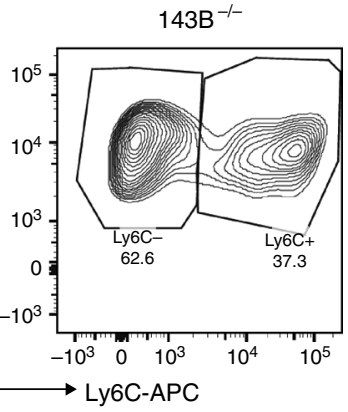

Ly6C-APC

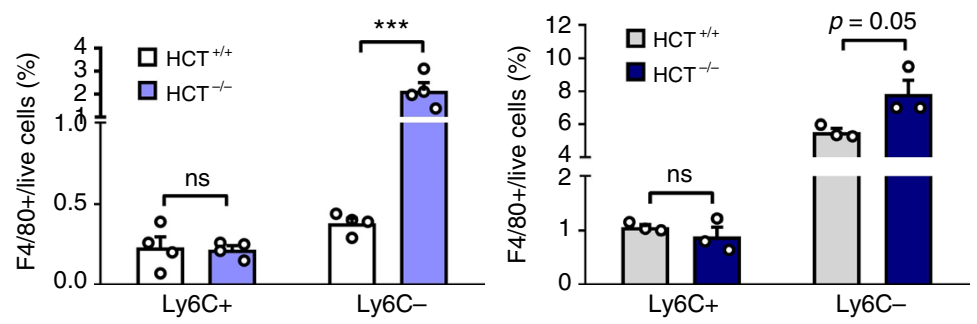

e

SMA DAPI

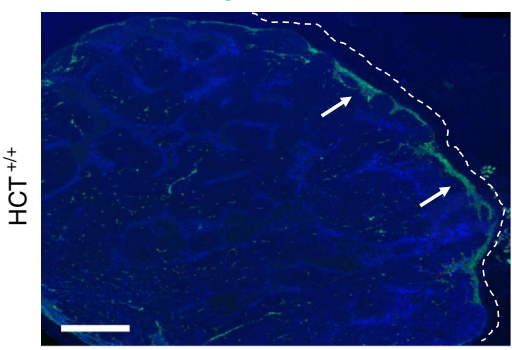

d
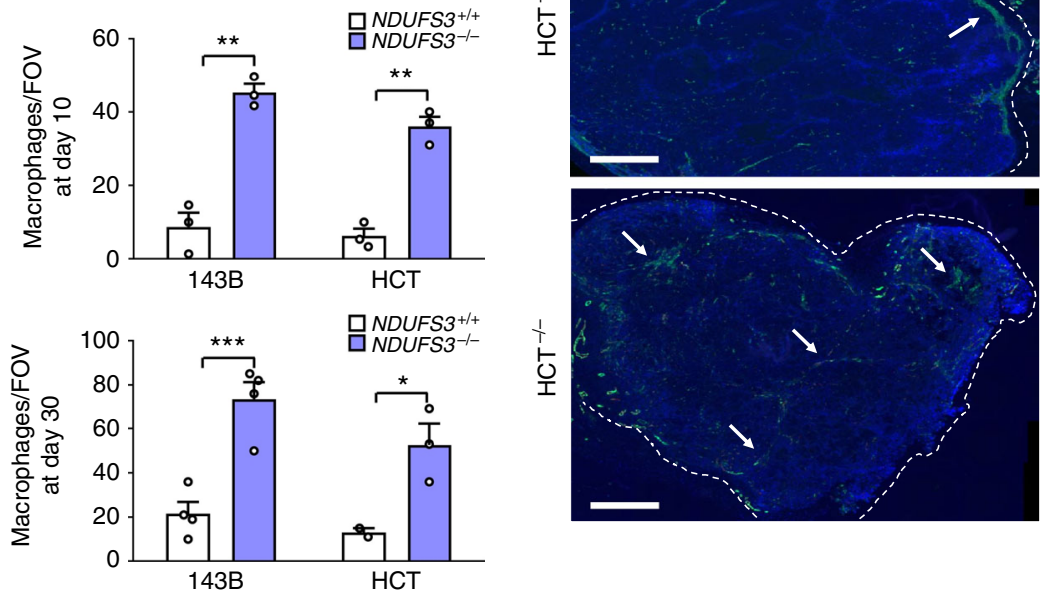

macrophages might contribute more than CAFs in progression of CI-deficient tumors. The prominent contribution of macrophages in association with CI-deficient cancer cells was furthermore observed in tumors in which the CI defect was due to high mutant loads of the m.3571insC/MT-ND1 mutation (Supplementary Fig. 15a), as well as in Drosophila lgl ${ }^{-1-} \mathrm{Ras}^{\mathrm{V} 12} \mathrm{V1}^{\mathrm{KD}}$ tumors where the macrophage component $(\mathrm{NimC} 1+$ ) of the hemocytes (the fly blood cells) was found increased compared to their controls
(Supplementary Fig. 15b). Additionally, the treatment of CIcompetent xenografts with metformin, besides reducing tumor growth at comparable levels with genetic CI KO (Supplementary Fig. 15c), led to a higher number of intra-tumor macrophages than their untreated controls, as well as to a higher number of CD11b + F4/80 + Ly6C - cells (Supplementary Fig. 15d, e). Thus, our data imply that targeting CI results in an increase of macrophages within the tumor, possibly in order to support survival. 
Fig. 6 Macrophage infiltration is a hallmark of $\mathrm{Cl}$-deficient tumor microenvironment. a Flow cytometry analysis of innate immune system populations in 143B $(n=4, \mathrm{df}=6)$ and $\mathrm{HCT}(n=5, \mathrm{df}=8)$ tumors. The contribution of macrophages $(\mathrm{F} 4 / 80+)[t(143 \mathrm{~B})=2.8, t(\mathrm{HCT})=2.8]$, neutrophils $($ Lys6G +$)$ $[t(143 B$, data were log-transformed $)=5]$, dendritic cells $(C D 11 c+F 4 / 80-)$ and natural killer cells $(C D 49 b+)$ is shown at day 30 post injection in ICRF nude mice. Data are mean + s.e.m. b Macrophage to cancer cell ratio in $143 \mathrm{~B}(n=4, \mathrm{df}=6, t=3.21)$ and $\mathrm{HCT}(n=5, \mathrm{df}=8, t=4.834)$ xenografts. Data are mean + s.e.m. Representative dot-plots display contribution of macrophages (F4/80 +, in blue) and neutrophils (Ly6G +, in red) among 100,000 acquired events (SSC-A/FSC-A plots) and their relative amount among CD45 + population (F4/80/Ly6G plots). All other cell populations are represented in gray. The gates indicate population percentages. c Flow cytometry analysis of undifferentiated (CD11b + Ly6C + ) and differentiated (CD11b + Ly6C - ) monocytes in 143B ( $n=4, \mathrm{df}=6, t=4.213)$ and $\mathrm{HCT}(n=3, \mathrm{df}=4, t=2.674)$ xenografts at day 30 post injection in ICRF nude mice. Representative contour-plots are shown. The gates indicate population percentages. Data are mean + s.e.m. $\mathbf{d}$ The number of macrophages (F4/80 + ) infiltrating tumor tissue counted per field of view (FOV) in xenografts at day $10[143 \mathrm{~B}(n=3, \mathrm{df}=4, t=8), \mathrm{HCT}(n=3, \mathrm{df}=4, t=9)]$ and at day $30[143 \mathrm{~B}(n=4, \mathrm{df}=6$, $t=5.376), \mathrm{HCT}(n=3, \mathrm{df}=3, t=3.201)$ ] post injection. Representative images of immunohistochemistry analysis for macrophage marker $\mathrm{F} 4 / 80$ in xenografts are shown. Dashed lines delineate the tumor margin. Scale bars: $50 \mu \mathrm{m}$. Data are mean + s.e.m. e Representative image of immunofluorescent staining of the SMA + microenvironment component in HCT xenografts. Dashed lines delineate the tumor margin. The arrows indicate the collective CAF formations. Scale bars: $50 \mu \mathrm{m}$. In each panel, statistical significance is specified with asterisks $\left({ }^{\star} p<0.05,{ }^{\star \star} p<0.01,{ }^{\star \star \star} p<0.001\right)$

Inhibiting TAMs increases efficacy of CI targeting. TAMs may exert either pro-inflammatory or pro-angiogenic functions during tumor progression, depending on whether they are polarized toward M1 or M2 population, respectively ${ }^{35}$. Since abnormal vascularization, comparable to the Endo + SMA - structures identified in CI-deficient cancers (Fig. 5a) has been associated with M2-mediated pro-angiogenic signals ${ }^{36}$, we analyzed CIcompetent and deficient xenografts for the markers generally associated with M1 (iNOS) or M2 (CD206, Arg1) macrophage populations. In all samples analyzed, the number of CD206expressing macrophages increased from day 10 to day 30, when more than $70 \%$ of TAMs were CD206 +, whereas the number of macrophages expressing iNOS was generally low in all samples analyzed $(10-20 \%$ in HCT and $1-4 \%$ in $143 \mathrm{~B})$, suggesting that the activation of protumorigenic TAMs is a property of both CIdeficient and control tumors (Supplementary Fig. 16a). Interestingly, iNOS expression, usually associated to M1 macrophages, was observed in CD206 ${ }^{\text {high }}$ and Arg1 ${ }^{\text {high }}$ population (Fig. 7a). There was no increase in the relative expression of any of the markers in CI-deficient tumors (Supplementary Fig. 16b), excluding the possibility that CI-deficient cancer cells are more capable to polarize TAMs to M2. Nonetheless, the total number of CD206 + and Arg1 expressing macrophages was higher in $143 \mathrm{~B}^{-1-}$ and $\mathrm{HCT}^{-1-}$ masses, whereas the number of iNOS expressing TAMs was either comparable or higher in CIcompetent tumors (Fig. 7b), showing that protumorigenic macrophages were generally more abundant in CI-deficient tumors. These data demonstrate that targeting CI did not potentiate M2 polarization, but was instead associated with higher macrophage abundance, in agreement with the increase in monocyte differentiation and higher macrophage infiltration identified in CIdeficient tumors (Fig. 6b-d).

Collectively, our data suggest that CI-deficient xenografts could rely on TAMs to promote survival. To prove this hypothesis, we depleted macrophages during CI-deficient tumor development. $\mathrm{HCT}^{-1-}$ xenografts were treated with liposomes containing clodronate, a bisphosphonate that induces selective apoptosis of phagocyting macrophages. Compared to vehicle-treated controls, mice receiving clodronate showed almost complete absence of xenograft growth (Fig. 7c). Despite the anti-tumorigenic effect of clodronate was observed also in $\mathrm{HCT}^{+/+}$tumors (Supplementary Fig. 17a), the treatment did not prevent $\mathrm{HCT}^{+/+}$cells from forming frank tumors, with histology similar to the untreated controls (Supplementary Fig. 17b). Instead, $\mathrm{HCT}^{-1-}$ xenografts treated with clodronate formed masses not larger than $10 \mathrm{~mm}^{3}$, which upon excision revealed to be white, indicating complete absence of vascularization (Fig. 7c). Similarly, when $143 \mathrm{~B}^{-1-}$ cells were injected in more permissive $R a g 1^{-I-} F V B / n$ mice, clodronate-treated tumors presented with a lower number of
SMAn-egative vessels (Supplementary Fig. 17c, d). Importantly, histology of clodronate-treated $\mathrm{HCT}^{-/-}$masses revealed extensive necrosis, normally absent from CI-deficient xenografts (Fig. 7c), demonstrating that CI-deficient cells depend on macrophages to form tumors. This finding has the potential clinical implication of enhancing metformin treatment in cancer, if combined with drugs blocking macrophages activity. Thus, we treated $\mathrm{HCT}^{+/+}$xenografts simultaneously with both metformin and colony stimulating factor 1 (CSF1) receptor inhibitor PLX3397, with the aim to specifically block CSF1-dependent macrophage infiltration in tumors. In line with the data from CI-deficient models, the combination of drugs significantly increased the efficacy of both PLX-3397 and metformin alone (Fig. 7d), proving that targeting macrophages is a promising approach to potentiate the effects of CI inhibitors in cancer, in a combinatorial regimen.

\section{Discussion}

Targeting CI is considered to be a promising anti-cancer therapeutic strategy, according to the bulk of data collected both in experimental settings $s^{1,37,38}$ and in clinical trials using metformin $^{2,39}$. This is indirectly supported by the negative selection of severe CI defects in human tumors, which in the rare occasions when they do occur, associate with the development of low-proliferative oncocytomas ${ }^{8}$. We here exploit a fine-tunable system of CI ablation in different cancer types to trigger conversion to oncocytomas, and demonstrate that HIF-1a destabilization is accountable for such indolent behavior. Nonetheless, the conflicting data regarding metformin efficacy $40-43$ and the fact that CI-deficient lesions continue to strive, point out the triggering of adaptive responses which our models allowed to dissect. In particular, we identified TAM-associated survival response in CI-deficient tumors and demonstrated that macrophage inhibition further decreased their tumorigenic potential.

Although HIF-1a destabilization has been previously described in CI-deficient cancer models, human oncocytomas, as well as in xenografts treated with metformin ${ }^{1,10,19}$, the molecular mechanisms linking these phenomena are still unclear. In particular, at least two players undergo significant changes in cancer cells deprived of $\mathrm{CI}$, converging toward the promotion of PHD activity and HIF-1a destabilization regardless of hypoxia, one of them being the accumulation of $\alpha-K^{30}$. Further, our results support the concept that OXPHOS alterations cause the redistribution of intracellular $\mathrm{O}_{2}$ from respiratory enzymes to $\mathrm{PHDs}^{31}$. In this metabolic frame of HIF-1a regulation, $\alpha-K G$ and $\mathrm{O}_{2}$ likely decrease the ability of CI-deficient cells to sense hypoxia, which is reflected in the hypoxic foci lacking nuclear HIF1 in CI-deficient tumors. 
a

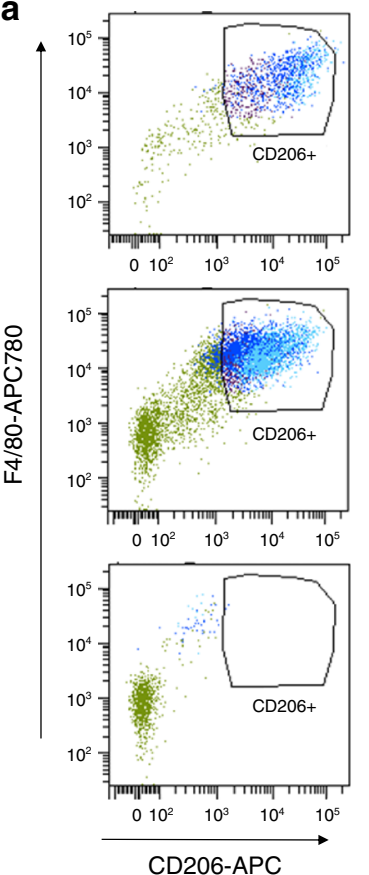

C

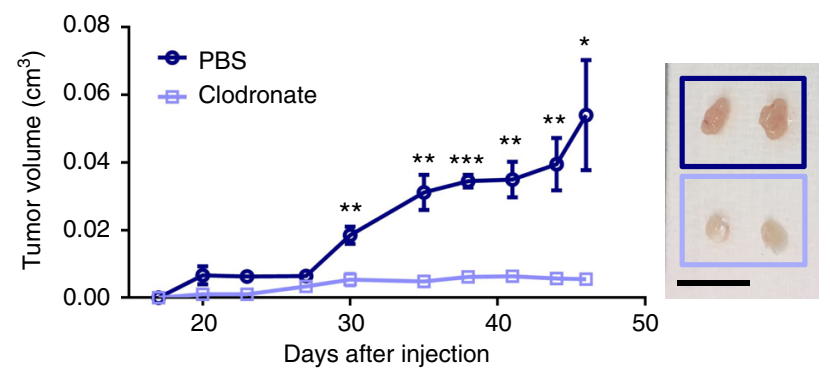

d

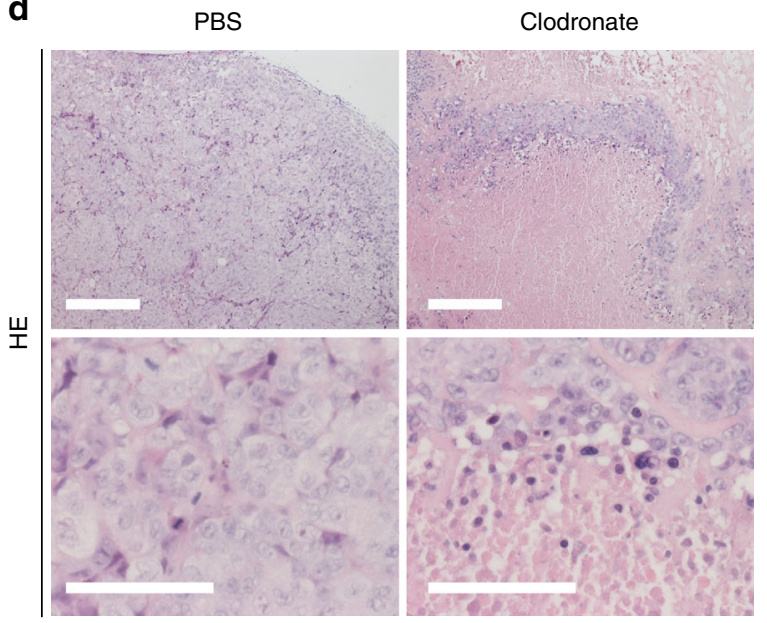

b

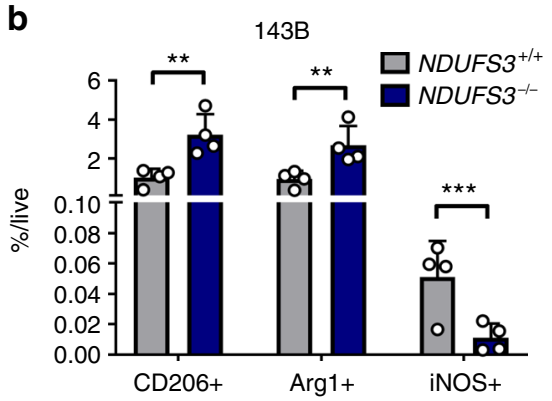

$\mathrm{HCT}^{-1-}$
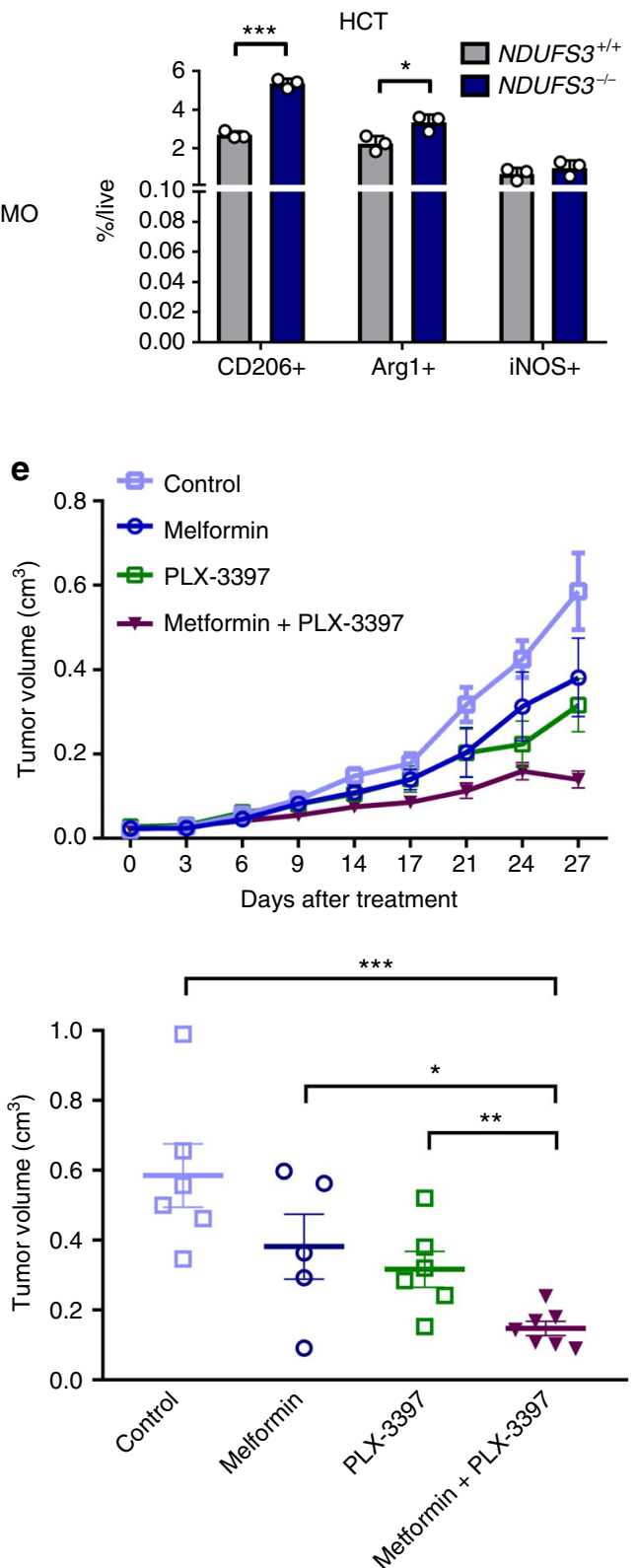

Due to the great number of pro-survival mechanisms activated by HIF1, it is reasonable to imply that the loss of HIF-1a challenges progression of CI-deficient masses. By complementing CI-deficient cells with TM-HIF-1 $\alpha$, we have provided proof for such concept. The consequences of HIF-1a destabilization in CIdeficient tumors are at least dual. As previously shown, it prevents the acquisition of a typical Warburg profile ${ }^{20}$, which is linked to the upregulation of several pro-glycolytic genes HIF1 is responsible to transcribe. More importantly, as demonstrated here, HIF1 inactivation impairs tumor vasculature in CI-null tumors, compromising their nutrient supply.

However, despite the loss of HIF-1 $\alpha$ and maintenance of a lowproliferative phenotype, CI-deficient tumor growth persists. In this respect, our observation of a different histological architecture in CI-deficient masses revealed that the striking abundance of microenvironment component in these tumors may be 
Fig. 7 TAMs support $\mathrm{Cl}$-deficient tumors growth and their inhibition enhances metformin effects. a Representative dot plots displaying macrophage populations expressing CD206, Arg1, and iNOS in $\mathrm{HCT}^{+/+}$and $\mathrm{HCT}^{-/-}$xenografts, and in fluorescence minus one (FMO) controls. iNOS (light blue) was set as the first front and Arg1 (dark blue) as the second front population, compared to CD206 (dark purple) and remaining immune cells (green). b Flow cytometry analysis of macrophage subpopulations markers CD206, Arg1, and iNOS in 143B and HCT tumors at day 30 post injection in ICRF nude mice. Statistics for 143B: $n=4, d f=6, t(C D 206)=3.756, t(\operatorname{Arg} 1)=3.219, t(i N O S)=3.143$. Statistics for HCT: $n=3, d f=4, t(C D 206)=14.96$, $t(\operatorname{Arg} 1)=3.348$. Data are mean + s.e.m. c Tumor growth curves of $\mathrm{HCT}^{-/-}$xenografts in ICRF nude mice treated with or without clodronate $[n=8$,

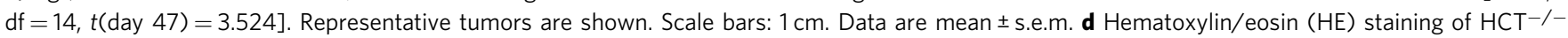
xenografts in ICRF nude mice treated with or without clodronate. Scale bars: $50 \mu \mathrm{m}$. e Tumor growth curves and volume at day 27 post-treatment of $\mathrm{HCT}^{-/}$- xenografts in CD-1 nude mice, treated with metformin ( $2 \mathrm{mg} \mathrm{mL}^{-1}$ in drinking water) or PLX-3397 (1.5 mg per day by oral gavage) alone and in combination [data were log-transformed, $n=5-7, \mathrm{df}$ (metformin versus double treatment) $=10, t$ (metformin versus double treatment) $=2.539$ ]. Data are mean \pm s.e.m. In each panel, statistical significance is specified with asterisks $\left({ }^{\star} p<0.05,{ }^{\star \star} p<0.01,{ }^{\star \star \star} p=0.001\right)$

accountable for their alternative, HIF-1a-independent nutrient supply. Even though this needs to be corroborated in a fully immunocompetent system, it is well-known that cells from the tumor microenvironment promote its progression ${ }^{44}$, and that the ultimate vascular phenotype of a neoplasm results from converging/synergistic cancer cell-autonomous and microenvironmentmediated pro-angiogenic signals ${ }^{32}$. Hence, abnormalized vessels typical of myeloid-driven vasculogenesis ${ }^{45}$, the infiltration of macrophages observed in CI-deficient tumors and the reduction of their number when HIF-1 $\alpha$ activity was complemented by TMHIF-1 $\alpha$, all together imply that macrophage abundance is a consequence of HIF-1a destabilization in CI-deficient tumors. This is furthermore supported by the fact that CI-deficient tumors display MIF downregulation, a phenomenon recently associated with macrophage-mediated vascularization activated as a compensatory response upon anti-VEGF treatment ${ }^{33}$. Indeed, since MIF is a known HIF1-responsive gene and CI targeting ultimately leads to lack of HIF1, our data indicate that TAM abundance may be a consequence of MIF downregulation. Intriguingly, MIF is a classical pro-survival cytokine, meaning that its downregulation may also contribute to the lower tumorigenic potential of the CI-deficient cancer cells. However, at the same time its downregulation paradoxically releases a microenvironment-mediated compensatory response, classifying MIF in the ever-growing group of double-edged oncojanus cancer genes $^{46}$. Considering the complexity of the cross-talk between cancer cells and microenvironment, we acknowledge that mechanisms apart from the HIF-MIF axis likely contribute to macrophage abundance in CI-deficient tumors. The role of CAFs needs to be further explored, since their secretion of extracellular matrix proteins supports macrophage migration, and CAFs also secrete their own pro-angiogenic factors ${ }^{32}$. It is interesting to note that repair-like patrolling macrophages $(\mathrm{CD} 11 \mathrm{~b}+\mathrm{Ly} 6 \mathrm{C}$ $-\mathrm{F} 4 / 80+$ ), we here identify as associated with CI-deficient cancers, have been described to promote angiogenesis and fibrosis in the wound ${ }^{47}$, meaning that their abundance in the context of cancer might explain the fibroblast contribution we observe in CI-deficient tumors.

Blocking TAM infiltration further reduced the tumorigenic potential of CI-deficient xenografts, as well as metformin-treated tumors, indicating that simultaneous targeting of CI and macrophages is an efficient synergistic approach to reduce tumor growth. Of note, CI inhibitors such as metformin and macrophage-targeting agents both exhibit cytostatic effects on cancer cells. Confining cancers in a low-proliferative state ought to be considered a valid alternative approach to cancer eradication, since turning cancer into a chronic disease slows down its genetic evolution and allows the pool of progressing cancer cells to competitively consume available resources ${ }^{48}$. Interestingly, both metformin and macrophage inhibitors are economical drugs, currently used in the clinics, with limited side effects when administered in diabetic and osteoporotic patients, respectively.
Overall, our results corroborate targeting CI as a valid anti-cancer strategy, although this may be overcome, at least in certain types of cancers, by macrophage-mediated adaptive response. We hence look forward to the clinical trials exploring the combinatorial effects of drugs that hit two such essential players of the adaptive process of cancer cells.

\section{Methods}

Cell lines and treatments. Osteosarcoma $143 \mathrm{~B} \mathrm{Tk}^{-}$cells and colorectal cancer HCT116 cells were used, both carrying wild-type mtDNA. 143B cells were purchased from ATCC (\#CRL-8303) and HCT116 cells were a kind gift from Prof. Paolo Pinton from the University of Ferrara. Cell origin was authenticated using AMPFISTRIdentifiler kit (Applied Biosystems \#4322288) and their STR profile corresponded to their putative background (Supplementary Fig. 1a). For basal conditions, cells were cultivated in Dulbecco's modified Eagle medium (DMEM) high glucose (Euroclone \#ECM0749L), supplemented with 10\% FBS (Euroclone \#ECS0180L), L-glutamine (2 mM, Euroclone \#ECB3000D), penicillin/ streptomycin $\left(1 \times\right.$, Euroclone \#ECB3001D), and uridine $\left(50 \mu \mathrm{gL}^{-1}\right.$,

Sigma-Aldrich \#U3003), in an incubator with a humidified atmosphere at $5 \% \mathrm{CO}_{2}$ and $37^{\circ} \mathrm{C}$. Cells were replaced by a fresh batch after 15 passages and mycoplasma testing was performed before disposal and after each thawing (approximately every 2 months). Experiments in hypoxia were performed using an Invivo2 300 (Baker Ruskinn) chamber, set at $5 \% \mathrm{CO}_{2}, 37^{\circ} \mathrm{C}$ and $1 \% \mathrm{O}_{2}$. Where indicated, cells were incubated for $3 \mathrm{~h}$ with dimethyloxalylglycine [DMOG $(1 \mathrm{mM})$, Sigma-Aldrich \#D3695] or MG132 (10 $\mu \mathrm{M}$, Sigma-Aldrich \#M7449) and $1 \mathrm{~h}$ with pimonidazole (100 $\mu \mathrm{M}$, Hypoxiprobe \#70132-50-3).

Genome editing for generation of NDUFS3 knockout. Plasmids containing CDNA of NDUFS3-targeted zinc finger endonucleases were purchased from SigmaAldrich (\#CKOZFND15168) and were used according to the manufacturer's instructions. Briefly, the plasmids were purified and transcribed in vitro using MessageMax caping kit (Cell Script \#C-MMA60710), Poly adenylation kit (Epicentre \#PAP5104H), and purified using MegaClear kit (Life Technologies \#AM1908). The pool of zinc finger endonucleases mRNAs (2.5 $\mu \mathrm{g}$ each) was transfected using Transit-mRNA transfection kit (Mirus \#2225) into 70\% confluent cells. Cells were split $48 \mathrm{~h}$ after transfection and DNA was extracted using Mammalian Genomic DNA Miniprep Kit (Sigma-Aldrich \#G1N350). Non-homologous repair efficiency was evaluated by Fluorescent PCR using KAPA2G Taq polymerase (Kapa Biosystems \#KK5601) with $58^{\circ} \mathrm{C}$ annealing and primers forward [Flc] CTGCCACAAGGAGCTAGGAC and reverse GCACAGGGAGATAAAAGGCA. Clonal selection was performed in order to identify the cells with frameshift NDFUS3 mutations. Selection media used for the single-cell growth in 96-well plates was composed of DMEM high glucose (Euroclone \#ECM0749L), supplemented with 20\% FBS (Euroclone \#ECS0180L), L-glutamine ( $2 \mathrm{mM}$, Euroclone \#ECB3000D), penicillin/streptomycin $(1 \times$, Euroclone \#ECB3001D), uridine $(50 \mu \mathrm{g}$ $\mathrm{mL}^{-1}$, Sigma-Aldrich \#U3003), L-Tryptophan (32 mg/L, Sigma-Aldrich \#T0254), and Nicotinamide (8 $\mathrm{mg} \mathrm{L}^{-1}$, Sigma-Aldrich \#72340). DNA extraction from 96well plates was performed using $8 \mu \mathrm{L}$ of Lysis Solution (Sigma-Aldrich \#L3289) and $80 \mu \mathrm{L}$ of Neutralization Buffer (Sigma-Aldrich \#N9784) per sample. Upon genotyping, heterozygous clones were first expanded, and subjected to a subsequent second transfection with NDUFS3 zinc finger pool. This step led to the selection of homozygous frameshift NDUFS3 mutants and homozygous wild-type revertants (Supplementary Fig. 1b). The latter were used as isogenic controls in all experiments described here. NDUFS 3 genotype was confirmed by Sanger sequencing using KAPA2G Taq polymerase (Kapa Biosystems \#KK5601) and Big Dye protocol (Life Technologies \#4337451). After the validation of single NDUFS3 ${ }^{-l-}$ clones, they were pooled for the experiments that followed (143B $n=5$, HCT $n=3)$.

Generation of cells with inducible NDUFS3 knockout. To create a stable transgenic cell line that re-expresses NDUFS 3 and allows its inducible knockout, the Retro-X Tet-Off Advanced Inducible Expression system (Clontech \#632105) 
was used by following manufacturer's instructions. First, $3 \times 10^{6}$ amphotrophic Phoenix (phxA) cells (ATCC ${ }^{\circledR}$ No. CRL-3213 ${ }^{\text {mII }}$ ) were transiently transfected with $20 \mu \mathrm{g}$ of pRetroX-Tet-Off Advanced vector and $0.9 \mu \mathrm{g}$ of helper plasmid using Lipofectamine 2000 (Thermo Fisher \#11668027). PhxA cells were maintained in the presence of transfection mix for $16 \mathrm{~h}$, then switched to DMEM for $24 \mathrm{~h}$, after which the retroviral supernatant was collected, filtered $(0.45 \mu \mathrm{m})$, supplemented with $8 \mu \mathrm{g} \mathrm{mL}^{-1}$ hexadimethrine bromide, and $2 \mathrm{~mL}$ was used to stably transfect $143 \mathrm{~B}$ cells $\left(50 \times 10^{3}\right)$ in 6 -well plates. The later were then (i) centrifuged 1800 r.p.m. at $32^{\circ} \mathrm{C}$ for $45 \mathrm{~min}$ and incubated at $32^{\circ} \mathrm{C}$ for $2 \mathrm{~h}$, (ii) replenished with fresh retroviral supernatants, re-centrifuged as indicated and incubated at $32{ }^{\circ} \mathrm{C}$ for additional $4 \mathrm{~h}$, (iii) replenished with normal medium and incubated overnight, (iv) replenished with $4 \mathrm{~mL}$ of fresh retroviral supernatant, centrifuged as indicated and incubated at $32{ }^{\circ} \mathrm{C}$ for $5 \mathrm{~h}$, (v) replenished with normal medium and incubated $48 \mathrm{~h}$, (vi) replenished with normal medium containing G418 (400 $\mu \mathrm{g} \mathrm{mL}^{-1}$, Sigma \#A1720) and incubated for $48 \mathrm{~h}$. The surviving cells were maintained in medium supplemented with $100 \mu \mathrm{g} \mathrm{mL}^{-1}$ G418. Single-cell cloning of selected cells was then performed and luciferase was transfected in each clone in order to identify cells with optimal transactivator activity. In particular, $2 \mu \mathrm{g}$ of pRetroX-Tight-Pur-Luc Control Vector was transiently transfected using X-tremeGENE HP DNA Transfection Reagent kit (Roche $\# 06366236001)$ in $143 \mathrm{~B}$ cells $\left(80 \times 10^{3}\right)$ seeded in 6 -well plates in the standard medium but containing Clontech tetracycline-free FBS. Each clone was then treated with/without Dox (100 ng mL ${ }^{-1}$, Sigma \#D9891) and after $48 \mathrm{~h}$, cells were harvested and processed according to the Dual-Luciferase Reported Assay System (Promega \#E1910) protocol. The luciferase activity was generally high in absence of Dox and low in cells cultivated with Dox medium. Five clones with lowest + Dox luciferase activity were pooled together and then the second viral transduction was performed as described above, using pRetroX-Tight-PurNDUFS3 vector. The selection of cells that uptaken the plasmid was performed using puromycin $\left(0.5 \mu \mathrm{g} \mathrm{mL}^{-1}\right.$, Sigma \#P8833). Single-cell cloning was performed to identify clones showing lack of NDUFS3 after Dox treatment and five clones were pooled to obtain $143 \mathrm{~B}^{-/-N D U F S 3}$ cell line.

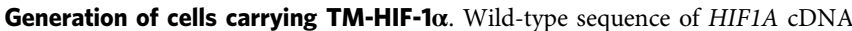
was cloned in pGEM (Promega \#A1360) vector and mutagenesis was performed using Quickchange Site-Directed Mutagenesis kit (Agilent \#200518), following manufacturer's instructions, to induce the mutations at the PHD hydroxylation sites following previously described indications ${ }^{24}$. In particular, three mutations were inserted in the HIF1A sequence, to substitute the prolyl hydroxylase (PHD)targeted prolines and Factor Inhibiting HIF (FIH)-targeted asparagine with residues that cannot be hydroxylated (P402A, P564G, and N803A). The triple mutant HIF1A was then transferred to pMSCV-Puro retroviral vector (Clontech \#PT33035 ). The empty vector and the vector containing TM-HIF-1a were then used to transduce 143B and HCT116 cells by following phxA transduction protocol described above. Cells carrying the vectors were selected with $2 \mu \mathrm{g} \mathrm{mL}^{-1}$ puromycin (Sigma \#P8833) and maintained in medium supplemented with $1 \mu \mathrm{g} \mathrm{mL}^{-1}$ puromycin. Clonal selection was performed to identify the clones with the highest TM-HIF-1 $\alpha$ protein levels in normoxia and a pool of 20 clones was made.

SDS-PAGE and western blot. Whole lysates of cultured cells and freshly snapfrozen xenograft samples were prepared in RIPA buffer (Tris-HCl pH 7.4 (50 $\mathrm{mM}), \mathrm{NaCl}(150 \mathrm{mM})$, SDS (1\%), Triton (1\%), EDTA pH $7.6(1 \mathrm{mM}))$ supplemented with protease inhibitors (Roche \#11873580001), and quantified by Lowry protein assay (Bio-Rad \#5000116). Samples were separated by SDS-PAGE and transferred onto nitrocellulose membrane using Turbo-pack system (Bio-Rad \#1704159SP5). Membranes were blocked $30 \mathrm{~min}$ at $37^{\circ} \mathrm{C}$ and incubated with primary antibodies using following dilutions/conditions: anti-NDUFS3 (AbCam \#177471) 1:1000/1-h at room temperature (RT); anti-HIF1a (GeneTex \#GTX127309) 1:2000/1-h at RT; anti-Vinculin (Sigma-Aldrich \#V9131) 1:10,000/ 1-h at RT; anti-pimonidazole (Hydroxyprobe \#4.3.11.3) 1:3000/2-h at $37^{\circ} \mathrm{C}$; antiHIF-1 $\alpha$-OH (Cell Signaling \#3434) 1:500/O/N at $4{ }^{\circ} \mathrm{C}$; anti-VDAC (AbCam \#154856) 1000/2-h at RT; anti-MT-CO2 (AbCam \#110258) 1:1000/2-h at RT; antiMIF (AbCam \#175189) 1:5000/1-h at RT; anti-ACTB (Santa Cruz \#SC-1615) 1:500/1-h at RT; anti-cleaved Caspase 3 (Cell Signaling Technology \#9661) 1:1000/ $\mathrm{O} / \mathrm{N}$ at $4{ }^{\circ} \mathrm{C}$; anti-PHD1 (Abcam \#ab108980) $1: 1000 / \mathrm{O} / \mathrm{N}$ at $4{ }^{\circ} \mathrm{C}$, anti-EGLN1/ PHD2 (Novus Bio \#NB100-137) 1:1000/O/N at $4^{\circ} \mathrm{C}$; anti-GAPDH (Sigma-Aldrich \#G8795) 1:20,000/2-h at RT. Washes were performed $4 \times 5$ min using TBS-Tween [0.1\% Tween 20 (Sigma-Aldrich \#P9416) in Tris Buffered Saline] and incubation with secondary antibodies (Jackson ImmunoResearch Laboratories \#111035144 and \#111035146), diluted 1:20,000 in TBS-Tween, was performed for $30 \mathrm{~min}$ at RT. Developing was performed by using Clarity Western ECL Substrate (Bio-Rad \#1705061) and exposing with ChemiDoc XRS ${ }^{+}$(Bio-Rad). For anti-HIF-1a-OH antibody, Western Breeze system (Life Technologies \#WB7106) was used for secondary antibody and developing solutions, following the manufacturer's instructions. Raw acquisition images are presented in Supplementary Fig. 18.

Mitochondrial-enriched fraction preparation. Mitochondria-enriched fractions were obtained by subcellular fractionation $\left(5-10 \times 10^{6}\right.$ cells $)$ in presence of digitonin $\left(50 \mu \mathrm{g} \mathrm{m}^{-1}\right)$. Crude mitochondria were obtained from $15-20 \times 10^{6}$ cells. The cell pellet was suspended in ice-cold buffer containing $200 \mathrm{mM}$ mannitol,
$70 \mathrm{mM}$ sucrose, $1 \mathrm{mM}$ EGTA, $10 \mathrm{mM}$ HEPES (pH 7.6) and mechanically disrupted with a glass/teflon Potter-Elvehjem homogenizer. Differential centrifugations $\left(600 \times g\right.$ for $10 \mathrm{~min}$ at $4{ }^{\circ} \mathrm{C}$ followed by $14,000 \times g$ for $10 \mathrm{~min}$ at $4{ }^{\circ} \mathrm{C}$ ) were performed to separate crude mitochondria from other subcellular fractions. Samples were stored at $-80^{\circ} \mathrm{C}$.

High-resolution clear native PAGE (hrCNE). For complex I in-gel activity (CI-IGA) assay and western blotting analysis, mitochondrial-enriched fractions were resuspended in mitochondrial buffer $(750 \mathrm{mM}$ aminocaproic acid, $50 \mathrm{mM}$ Bis-Tris, $\mathrm{pH}=7)$ and solubilized by adding DDM/protein ratio of $2.5(\mathrm{~g} / \mathrm{g})$. Suspension was incubated on ice for $10 \mathrm{~min}$ and then centrifuged at $13,000 \times \mathrm{g}$ for $15 \mathrm{~min}$. Aliquots of supernatants $(80 \mu \mathrm{g}$ protein) were separated by $4-16 \%$ first dimension hrCNE gradient gel (NativePAGE ${ }^{\mathrm{mm}} 4-16 \%$ Bis-Tris Protein Gels, Invitrogen, \#BN1002BOX) using as anode buffer $25 \mathrm{mM} \mathrm{pH}=7$ and as cathode buffer $50 \mathrm{mM}$ Tris, $7.5 \mathrm{mM}$ Imidazole, $0.02 \% \mathrm{n}$-Dodecyl $\beta$-D-maltoside (Sigma-Aldrich \#D4641), 0.05\% sodium deoxycholate (Sigma-Aldrich, \#D6750). Proteins were either transferred onto a nitrocellulose membrane at $100 \mathrm{~V}$ for $1 \mathrm{~h}$ at room temperature or complex I in-gel activity (CI-IGA) assay was performed. Briefly, gel was rinsed in cathode buffer and incubated at room temperature for $15 \mathrm{~min}$ in a solution containing $2 \mathrm{mM}$ Tris $\mathrm{pH}=7.4,0.5 \%$ 3-(4,5-Dimethyl-2-thiazolyl)-2,5diphenyl-2H-tetrazolium bromide (MTT, Sigma-Aldrich, \#M5655), $0.02 \%$ reduced $\beta$-Nicotinamide adenine dinucleotide (NADH, Sigma-Aldrich, \#N8129) under constant agitation and protected from light.

Measurement of ATP synthesis rate. The rate of mitochondrial ATP synthesis driven by CI, CII, and CIII was measured in digitonin-permeabilized cells. After trypsinization, cells $\left(10 \times 10^{6} \mathrm{~mL}^{-1}\right)$ were suspended in a buffer containing $150 \mathrm{mM} \mathrm{KCl}, 25 \mathrm{mM}$ Tris-HCl, $2 \mathrm{mM}$ EDTA (ethylenediaminetetraacetic acid), $0.1 \%$ bovine serum albumin, $10 \mathrm{mM}$ potassium phosphate, $0.1 \mathrm{mM} \mathrm{MgCl}_{2}, \mathrm{pH} 7.4$, kept at room temperature for $15 \mathrm{~min}$, then incubated with $50 \mu \mathrm{g} \mathrm{mL}^{-1}$ digitonin until $90-100 \%$ of cells were positive to Trypan Blue staining. Aliquots of $3 \times 10^{5}$ permeabilized cells were incubated in the same buffer in the presence of the adenylate kinase inhibitor $\mathrm{P}^{1}, \mathrm{P}^{5}$-di(adenosine- $\left.5^{\prime}\right)$ pentaphosphate $(0.1 \mathrm{mM})$ and OXPHOS complexes substrates, chemiluminescence was determined as a function of time with Sirius L Tube luminometer (Titertek-Berthold, Pforzheim, Germany). The chemiluminescence signal was calibrated with an internal ATP standard after the addition of $10 \mu \mathrm{M}$ oligomycin. The rates of the ATP synthesis were normalized to protein content and citrate synthase (CS) activity ${ }^{49}$.

Glucose consumption. Glucose consumption was determined by using the glucose oxidase (GO) assay kit (Sigma-Aldrich \#GAGO20) scaling down the manufacturer protocol to a final volume of $1 \mathrm{~mL}$. Briefly, cells were seeded in 6-well plates $\left(1 \times 10^{5}\right.$ cells/well $)$ in high glucose medium. After $48 \mathrm{~h}$, cells were washed in PBS and incubated with DMEM-high glucose without phenol red. Aliquots of $100 \mu \mathrm{L}$ of medium were taken at time 0 and after $24 \mathrm{~h}$ and $48 \mathrm{~h}$ of incubation and $3 \mu \mathrm{L}$ of samples were used to determine the glucose concentration, by the enzymatic reaction of glucose oxidase coupled to peroxidase-mediated oxidation of reduced o-Dianisidine $\left(\lambda=540 \mathrm{~nm}, 30 \mathrm{~min}, 37^{\circ} \mathrm{C}\right)$. The data obtained were normalized on cell number.

Lactate production. Cells were seeded in 6-well plates $\left(3 \times 10^{5}\right.$ cells/well $)$ in $2 \mathrm{~mL}$ of high-glucose medium. After $24 \mathrm{~h}$ and $48 \mathrm{~h}$, aliquots of medium were collected and de-proteinated with $6 \%$ perchloric acid, vortexed and incubated in ice for $1 \mathrm{~min}$. Samples were centrifuged at 13,000 r.p.m. at $4^{\circ} \mathrm{C}$ for $2 \mathrm{~min}$ and the lactate concentration in supernatants was determined by measuring NADH $(\lambda=340 \mathrm{~nm}$; $\varepsilon=6.22 \mathrm{mM}^{-1} \mathrm{~cm}^{-1}$ ) production in a buffer containing $320 \mathrm{mM}$ glycine, $320 \mathrm{mM}$ hydrazine, $2.4 \mathrm{mM} \mathrm{NAD}^{+}$and $2 \mathrm{U} \mathrm{mL}^{-1} \mathrm{~L}$-lactic dehydrogenase (Sigma-Aldrich \#L1006) after $30 \mathrm{~min}$ of reaction at $37^{\circ} \mathrm{C}$. Data were expressed as pmoles of lactate produced per cell.

Oxygen consumption rate. Mitochondrial respiration was evaluated using the Seahorse XFe Cell Mito Stress Test Kit (Seahorse Bioscience \#103015-100) following the manufacturer instructions. Cells were seeded $\left(3 \times 10^{4}\right.$ cells/well $)$ into XFe24 cell culture plate and allowed to attach for $24 \mathrm{~h}$. Cell culture media was replaced with XF media (Seahorse Bioscience \#103334-100). OCR was measured over a $3 \mathrm{~min}$ period, followed by $3 \mathrm{~min}$ of mixing and re-oxygenation of the media. For Mito Stress Test, complete growth medium was replaced with $670 \mu \mathrm{L}$ of unbuffered XF media supplemented with $10 \mathrm{mM}$ glucose $\mathrm{pH} 7.4$ pre-warmed at $37^{\circ} \mathrm{C}$. Cells were incubated at $37^{\circ} \mathrm{C}$ for $30 \mathrm{~min}$ to allow temperature and $\mathrm{pH}$ equilibration. After an OCR baseline measurement, $70 \mu \mathrm{L}$ of oligomycin, carbonyl cyanide-p-trifluoromethoxyphenylhydrazone (FCCP), and rotenone plus antimycin A were sequentially added to each well to reach final concentrations of $1 \mu \mathrm{M}$ oligomycin, $0.25 \mu \mathrm{M}$ FCCP, and $1 \mu \mathrm{M}$ rotenone and antimycin A. Three measurements of OCR were obtained following injection of each drug and drug concentrations optimized on cell lines prior to experiments. At the end of each experiment, the medium was removed and SRB assay was performed to determine the amount of total cell proteins as described above. OCR data were normalized to total protein levels (SRB protein assay) in each well. Each cell line was represented 
in five wells per experiment ( $n=3$ replicate experiments). Data are normalized on $\mathrm{SRB}$ absorbance and expressed as pmoles of $\mathrm{O}_{2}$ per minute.

ATP content measurement. Total ATP content was determined by using the ATPlite Luminescence Assay System (Perkin Elmer \#6016943). Cells (3-1.5 × 104) were seeded in a 96-wells plate in high glucose DMEM. After $24 \mathrm{~h}$, cells were washed twice with PBS and incubated with high glucose DMEM or with galactose DMEM. ATP content was measured after $16 \mathrm{~h}$ following the manufacturer protocol. Luminescence was detected using a multilabel counter Victor3 (Perkin Elmer). Data are normalized on cell number detected by SRB assay.

\section{Evaluation of Krebs-cycle metabolite concentrations. Absolute metabolite} concentrations were measured with Carcinoscope analysis [Human Metabolome Technologies (HMT)] that uses capillary electrophoresis coupled to time of flight/ triple quadrupole mass spectrometry. Metabolites were extracted using 100\% methanol supplemented with $550 \mu \mathrm{L}$ of internal standard solution provided by HMT from 2 to 5 million cells seeded on $90 \mathrm{~mm}$ plate. The metabolite concentrations of in vitro cell preparations were normalized to the number of viable cells. Tumor samples were grinded in liquid nitrogen before proceeding with the analysis. The measurement was corroborated by LC-based metabolite quantification at the Metabolomics Core Technology Platform of the Excellence cluster CellNetworks (University of Heidelberg; grant no. ZUK 49/2010-3002962).

Citrate ${ }^{13} \mathrm{C}$ isotopomer analysis. For mass isotopomer analysis, cells were incubated for $3 \mathrm{~h}$ either with DMEM high glucose (Euroclone \#ECM0749L) supplemented with $\left[{ }^{13} \mathrm{C}\right]$ labeled glutamine $(2 \mathrm{mM})$ or with DMEM (Life Technologies \#11966-025) supplemented with sodium pyruvate $\left(110 \mathrm{mg} \mathrm{L}^{-1}\right.$, Sigma-Aldrich \#P2256) and $\left[{ }^{13} \mathrm{C}\right]$ labeled glucose $(25 \mathrm{mM})$. Nutrients labeled with ${ }^{13} \mathrm{C}$ were purchased from Cambridge Isotope Laboratories (\#CLM-1396 and \#CLM-1822). Metabolite extraction was performed as described above and the isotopomer distribution was evaluated with F-Scope analysis (Human Metabolite Technologies).

Reactive oxygen species measurement. To determine the $\mathrm{H}_{2} \mathrm{O}_{2}$ production, cells were incubated with $2 \mu \mathrm{M}$ 2,7-dichlorodihydrofluorescein diacetate $\left(\mathrm{H}_{2} \mathrm{DCFDA}\right)$ (Life Technologies \#D399) added to 100,000 cells, for $30 \mathrm{~min}$ at $37^{\circ} \mathrm{C}$. The cells were then collected and the reaction was stopped by placing them in an ice bath for $5 \mathrm{~min}$. The cells were disrupted by treatment with Triton X-100 (2\%) and centrifuged at $2500 \times g$ for $20 \mathrm{~min}$ at $4{ }^{\circ} \mathrm{C}$. The supernatant was used to measure fluorescence emission (excitation, $485 \mathrm{~nm}$; emission, $535 \mathrm{~nm}$ ) using a multilabel counter Victor3 (Perkin Elmer, Turku, Finland). The amount of $\mathrm{H}_{2} \mathrm{O}_{2}$ produced was calculated by using a standard curve of $2,7-\mathrm{DCFH}_{2}$ in which $1 \mu \mathrm{M}$ of 2,7 $\mathrm{DCFH}_{2}$ represented $1 \mu \mathrm{M}$ of $\mathrm{H}_{2} \mathrm{O}_{2}{ }^{50}$.

3D colony growth assay. Tumor cells were seeded in 24 -well dishes at 500 cells/ dish in $150 \mu \mathrm{L}$ of 2:1 Rat tail collagen-I (Corning \#354249) and Matrigel (Corning \#356234), yielding a final collagen concentration of $4 \mathrm{mg} \mathrm{mL}^{-1}$ and a final Matrigel concentration of $2 \mathrm{mg} \mathrm{mL}^{-1}$. The wells were pre-coated with $50 \mu \mathrm{L}$ of collagen/ matrigel media. The colonies were maintained in DMEM in basal conditions and analyzed after 14 days. For data in Fig. $1 \mathrm{~b}$ the invasive margins of the colonies were counted in 10 random colonies per sample $(n=3)$. For data in Supplementary Fig. $8 c$, colony number and average diameter were evaluated in 10 random colonies per sample $(n=6)$.

Xenograft growth. For in vivo studies, $n u / n u$ mice (CD- $1^{\oplus}$ Nude Mouse Crl:CD1Foxn $1^{\mathrm{n}}$ ) were purchased from Charles River Laboratories. Where indicated, ICRF nude or Rag1 ${ }^{-1-} \mathrm{FVB} / \mathrm{n}$ mice available at The Francis Crick Institute Biological Research Facility were used. We have complied with all relevant ethical regulations for animal testing and research. The animals were treated according to institutional guidelines and regulations at Paracelsus Medical University Salzburg, The Francis Crick Institute and University of Bologna. Respectively, the study received ethical approval from the Salzburg State Ethics Research Committee (20901TVG/112), UK Home Office (project license PPL number P83B37B3C) and Italian Ministry of Health (authorization code 437/2018-PR). Five to six-week-old female mice were subcutaneously injected with a $100 \mu \mathrm{L}$ suspension of $5 \times 10^{6}$ cells in serum free medium and matrigel (Corning \#356234) in the right flank of the animal. For experiments in Figs. 3g, 7c and Supplementary Fig. 17a-d, a bilateral inoculation was performed. Xenograft size was measured with a sliding caliper twice a week, according to the formula: volume $=$ width $\times$ height $\times$ length $/ 2$. Mice were sacrificed either simultaneously, when the first xenograft reached $10 \%$ of animal weight (Fig. 1a, f, Fig. 3g, Fig. 7c, Supplementary Fig. 8d, Supplementary Fig. 15c and Supplementary Fig. 17a-d), or consecutively, when each animal reached xenograft volume corresponding to $10 \%$ of animal weight or met the termination criteria (Fig. 2a and Fig. 7e). For the Dox-induced experiment, 3\% sucrose with or without Dox $\left(1 \mathrm{mg} \mathrm{mL}^{-1}\right)$ was added into the drinking water of the mice, which were sequentially randomized in Dox-treated and control group when tumor would reach $450 \mathrm{~mm}^{3}$. For CSF1-R inhibition, PLX-3397 (Pexidartinib, Apex BioTechnology \#B5854) was dissolved in DMSO and suspension was made by dilution in the aqueous solution of $0.5 \%$ hydroxypropyl methyl cellulose (HPMC, Sigma
\#H7509) and 1\% polysorbate (PS80, Sigma \#59924). The drug (1.5 mg per mouse) was administered by oral gavage for 20 consecutive days with $100 \mu \mathrm{L}$ of suspension. Metformin was added to drinking water at concentration of $2 \mathrm{mg} \mathrm{mL}^{-1}$. Fresh water/metformin pouches were provided twice weekly. The PLX-3397 and/or metformin treatment was started when a tumor would reach $50 \mathrm{~mm}^{3}$. Prior to the sacrifice ( $3 \mathrm{~h}$ before) all animals were injected intraperitoneally with pimonidazole (60 mg kg-1, Hypoxyprobe \#70132-50-3) diluted in saline solution. For the clodronate treatment experiments in Fig. $7 \mathrm{c}$ and Supplementary Fig. 17a-d, the animals were pre-injected intraperitoneally with PBS or clodronate liposomes $(100 \mu \mathrm{L}$, ClodronateLiposomes, Liposoma BV) on the day prior to cell injection. On the day of tumor cell injection, $5 \times 10^{6}$ cells in growth factor reduced matrigel $(100 \mu \mathrm{L})$ were injected subcutaneously, immediately followed by injection of $40 \mu \mathrm{L}$ of PBS or clodronate liposomes at the same position. The mice continued to receive intraperitoneal injection of liposomes twice weekly $(100 \mu \mathrm{L})$.

Immunohistochemical staining. The samples were formalin fixed following standard protocols. Tissue sections $(4 \mu \mathrm{m})$ were deparaffinized in xylene, rehydrated in absolute 2-propanol followed by heat-induced epitope retrieval in TE-T buffer (10 mM Tris pH 8.0, $1 \mathrm{mM}$ EDTA, $0.05 \%$ Tween 20) for $40 \mathrm{~min}$ at $95^{\circ} \mathrm{C}$ and $20 \mathrm{~min}$ at RT. Sections were equilibrated with phosphate-buffered saline containing $0.5 \%$ Tween 20 (PBS-T pH 7.4). Primary antibodies were diluted in Antibody diluent with background reducing components (Dako \#S3022) and incubated at RT for 30 min. Blocking, secondary antibodies staining and development were carried out using the Envision Detection System (Dako \#K4007 and \#K4011) according to the manufacturer's instructions. Slides were counterstained with hematoxylin. The following primary antibodies were used: rabbit monoclonal antiNDUFS3 (1:200, Abcam \#177471); rabbit polyclonal anti-HIF-1a (1:350, SigmaAldrich \#HPA001275); mouse monoclonal anti-pimonidazole (1:400, Hypoxyprobe \#Mab-4.3.11.3); rabbit monoclonal anti-CD-31 (1:50; Abcam \#28364); mouse monoclonal anti-KI-67 (1:100, Dako \#M7240); mouse monoclonal anti-MT-CO1 (1:1000, Abcam \#14705); mouse monoclonal anti-NDUFS4 (1:1000, Abcam \#55540), and rat monoclonal F4/80 (1:100, eBiosciences \#14-4801). Neutrophil marker 2 b10 antibody was developed in house at The Francis Crick Institute. For macrophage staining with anti-F4/80, trypsin-based antigen retrieval $(0.05 \%$ trypsin in $0.1 \mathrm{mM}$ Calcium chloride solution, $\mathrm{pH} 7.8$ ) was performed for $30 \mathrm{~min}$ at $37^{\circ} \mathrm{C}$, and biotinylated goat anti-rat secondary antibody (Sigma-Aldrich \#A9037) was used, together with VECTASTAIN ABC-HRP Kit (Vector Laboratories \#PK4005). Hematoxylin/eosin and Masson's trichrome staining were performed on $4 \mu \mathrm{m}$ sections following standard protocols. For evaluation of KI-67 positive nuclei, cells were counted at $\times 20$ magnification in 5-10 fields of view per tumor. Macrophages $(\mathrm{F} 4 / 80+)$ were counted at of $\times 20$ magnification in three fields of view per tumor.

Electron microscopy. Samples were fixed using paraformaldehyde (2\%) and glutaraldehyde $(2.5 \%)$ in cacodylate buffer $(0.1 \mathrm{M})$ for $24 \mathrm{~h}$ and then washed, kept in the cacodylate buffer at $+4{ }^{\circ} \mathrm{C}$, fixed with glutaraldehyde $(2.5 \%)$ and $1 \%$ osmium tetroxide, dehydrated in alcohol and propylene oxide and embedded in Epon 812 Sections $(1 \mu \mathrm{m})$ were stained with $1 \%$ toluidine blue for morphology control and electron microscopy area selection. The sections were observed with JEM-1011 transmission electron microscope (JEOL Ltd). At least two different areas were observed for each tumor. Mitochondrial morphology was evaluated by measuring short mitochondrial axis and counting cristae in 50 mitochondria per sample.

Quantitative real-time PCR. Gene expression was analyzed following minimal information for publication of Quantitative Real-Time PCR (qRT-PCR) Experiments guidelines ${ }^{51}$. In particular, RNA was extracted using Trizol (Life Technologies \#15596018) for cell lines, and Mammalian Genomic DNA Miniprep Kit (Sigma-Aldrich \#RTN70) for snap-frozen xenograft samples. High Capacity cDNA Reverse Transcription Kit (Applied Biosystems \#4368814) was used for preparation of cDNA with random hexamers starting from $300 \mathrm{ng}$ of RNA. Primer sequences were designed using Primer3 software ${ }^{52}$. The presence of $3^{\prime}$ intra/inter primer homology was ruled out using IDT OligoAnalyzer tool (http://eu.idtdna.com/ analyzer/Applications/OligoAnalyzer/) and the availability of the target sequence was evaluated by prediction of the cDNA secondary structure using Mfold web server $^{53}$. Primer sequences are reported in Supplementary Table 1. The PCR reaction was performed with GoTaq qPCR Master Mix (Promega \#A6002) and run in 7500 Fast Real-Time PCR System (Applied Biosystems), using following conditions: $95^{\circ} \mathrm{C} 5 \mathrm{~min} ; 45$ cycles of $95^{\circ} \mathrm{C} 15 \mathrm{~s}$ and $60^{\circ} \mathrm{C} 45 \mathrm{~s}$. The calculations were performed following $2^{-\Delta \Delta \mathrm{CT}}$ method [CT(control)-CT(experiment)], where the control was calculated as the average CT value deriving from control samples. The normalization was performed using TBP. The statistical significance was calculated using the $\triangle \mathrm{CT}$ values [CT(gene of interest)-CT(reference gene)] for each biological replicate in a group and applying Student's $t$-test ${ }^{54}$. Primer sequences are available in Supplementary Table 1.

Fly lines and treatments. Fly lines: yw, hs-Flp; l(2) $\mathrm{gl}^{4} / \mathrm{CyO}$; UAS-luc $\mathrm{KD}_{-} \mathrm{yw}$, hs-Flp; l(2)gl ${ }^{4} / \mathrm{CyO}$; UAS-NDUFV1 ${ }^{\mathrm{KD}}$-w; l(2)gl ${ }^{4} / \mathrm{CyO}$; act:CD2:Gal4, UAS-GFP/ TM6b-w; l(2)gl4, FRT40A/In(2LR)GlaBc; UAS-Ras V12, UAS-luc ${ }^{\mathrm{KD}} / \mathrm{TM} 6 \mathrm{~b}-\mathrm{w}$;

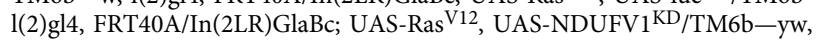


hs-flp, tub-Gal4, UAS-GFP; tub-Gal80, FRT40A. For experiments in Fig. 2f, larvae of the right genotypes were selected at $144 \pm 2 \mathrm{~h}$ development, transferred in a $1.5 \mathrm{~mL}$ vial plugged with foam and immersed for $2 \mathrm{~min}$ in a water bath at $37^{\circ} \mathrm{C}$. After the heat-shock, larvae were immediately transferred onto fresh food and allowed to grow for additional $48 \mathrm{~h}$ before dissection. For experiments in Supplementary Fig. $5 \mathrm{c}$ and Supplementary Fig. 9e, larvae were heat-shocked at $48 \pm 4 \mathrm{~h}$ development for $10 \mathrm{~min}$ in a water bath at $37^{\circ} \mathrm{C}$ and allowed to grow for additional $72 \mathrm{~h}$ before dissection. All the experiments were carried out at $25^{\circ} \mathrm{C}$. In all the control experiments, the UAS-luc ${ }^{\mathrm{KD}}$ line was used as an irrelevant dsRNA. For validation of the $\mathrm{VI}^{\mathrm{KD}}$ construct, $\mathrm{UAS}-\mathrm{VI}^{\mathrm{KD}}$ flies were crossed with act-Gal4 flies; the progeny was collected at the end of the larval life and processed as to obtain the total RNA for a qRT-PCR analysis.

Drosophila disc isolation and volume calculation. For experiments in Fig. $2 \mathrm{f}$, $192 \pm 2$ h larvae displaying GFP ${ }^{+}$cells were selected under a Nikon SMZ1000 fluorescence stereoscope. Collected larvae were dissected in cold PBS, tumorous imaginal wing discs were isolated and photographed. Major and minor axes were measured for each wing disc with Image (NIH) and volumes were calculated approximating disc shape to a spheroid with depth $=$ width. A minimum of 25 discs were analyzed for each replicate.

Immunofluorescent staining. For xenograft analysis, the samples were formalin fixed following standard protocols. Tissue sections $(4 \mu \mathrm{m})$ were deparaffinised in xylene, rehydrated in absolute 2-propanol followed by citrate antigen retrieval ( $10 \mathrm{mM}$ sodium citrate, $\mathrm{pH}$ 6) for $15 \mathrm{~min}$ at $95^{\circ} \mathrm{C}$ and $20 \mathrm{~min}$ at RT. Blocking was performed with goat serum (Abcam \#156046) for $10 \mathrm{~min}$ at RT and incubation with Alexa Fluor secondary antibodies (488-goat anti-mouse diluted 1:500 and 555-goat anti-rat diluted 1:350) for $40 \mathrm{~min}$ at RT. The following primary antibodies were used: rat anti-Endomucin (1:200, Santa Cruz \#SC-65495) and mouse antiSMA (1:750, Dako \#M0851). Slides were mounted with Vectashield Antifade Mounting Medium with DAPI (Vector Laboratories, \#H-1200). Vessel size was evaluated by measuring the longer diameter of 30 endomucin positive cells per tumor and avoiding areas of collective fibroblast infiltration. Fibroblasts $\left(\mathrm{SMA}^{+} \mathrm{Endo}^{-}\right)$and immature vessels $\left(\mathrm{Endo}^{+} \mathrm{SMA}^{-}\right)$were counted in five fields of view at $\times 20$ magnification per tumor. Images were taken with Zeiss Axio Scope. Z1 scanner using ZEN software (Carl Zeiss Microscopy GmbH, Germany). For pimonidazole staining, $8 \mu \mathrm{m}$ sections of snap-frozen samples were prepared and FITC-conjugated mouse monoclonal anti-pimonidazole antibody (1:400, Hypoxyprobe \#Mab-4.3.11.3) was used following manufacturer's indications. In vitro immunofluorescence for anti-HIF-1a (1:350, Sigma-Aldrich \#HPA001275) was performed using manufacturer's indications. Images for pimonidazole and HIF-1a staining were taken with an inverted Nikon Eclipse Ti-U epifluorescence microscope, using Metamorph software (Universal Imaging). For Drosophila experiments, tissues isolated from selected larvae were fixed and stained according to standard protocols. Primary antibodies: rabbit anti-cleaved caspase 3 (1:100, Cell Signaling \#9961), mouse anti-MMP1 (1:50, DSHB, Iowa University), rabbit antiSima/HIF-1 $1 \alpha^{55}$, mouse anti-NimrodC1 (original antibody by Istvan Andó sent by Julia Cordero), rabbit polyclonal anti-phospho-histone H3 (Ser10) (1:100, Cell Signaling Technology \#9701). Secondary antibodies: anti-mouse 555 Alexa Fluor (1:200) and anti-rabbit Cy5 DyLight (Jackson Laboratories, 1:500). Confocal images were processed as a whole with Adobe Photoshop. All the images shown represent a single confocal stack. Clone area, clone roundness and macrophage area were calculated by using ImageJ (NIH). For clone area (Fig. 2f) and roundness (Supplementary Fig. 5c), 20 random clones from separate discs were imaged. For cell density and caspase 3 (Supplementary Fig. 5b) quantification, marked cells present in four areas of $100 \times 100 \mu \mathrm{m}$ captured at different focal planes were counted for each tumor, for a total of 6 and 10 tumors, respectively.

Flow cytometry. Cell isolation was performed as previously described ${ }^{56}$. Briefly, xenograft samples $\left(\sim 50 \mathrm{~mm}^{3}\right)$ were digested immediately after the sacrifice for 40 min at $37^{\circ} \mathrm{C}$ with Liberase TL (Sigma \#5401020001), Liberase TM (Sigma \#5401135001) and DNaseI (Sigma \#DN25) in HBSS and passed through a $100 \mu \mathrm{m}$ strainer. Hypotonic lysis with Red Blood Cell Lysis Buffer (Sigma \#11814389001) was performed and remaining cells were washed with MACS buffer (2 mM EDTA, 0.5\% BSA in PBS), blocked using FcR Blocking Reagent (Miltenyi \#130-092-575) and incubated with panels of pre-labeled antibodies. In parallel, spleen, lung and a control tumor tissue were digested together and stained for fluorescence minus one (FMO) reading which was considered while setting the gating strategy. Following panels were used: Panel 1 (for discrimination of non-cancer versus cancer cells, and for the analysis of the immune cell/fibroblast contribution in the tumor): antiCD298-APC (clone LNH-94, Biolegend \#341706), anti-CD45-APC780 (clone 30F11, eBioscience \#47-0451-80), and anti-CD31-PeCy7 (clone 390, eBioscience \#250311-82); Panel 2 (for analysis of the tumor macrophage, neutrophil, natural killer cell and dendritic cell contribution): anti-anti-CD45-PE (clone 30-F11, eBioscience \#12-0451-82), anti-CD11b-ef450 (clone M1/70, eBioscience \#48-0112-82), anti-F4/ 80-APC780 (clone BM8, eBioscience \#47-4801-80), anti-Ly6G-APC (clone 1A8, BD Bioscience \#560599), anti-CD11c-PeCy7 (clone N418, Biolegend \#117317), and anti-CD49b-FITC (clone 30-F11, Biolegend \#108905). Panel 3 (for analysis of M1/M2 protumorigenic macrophages): anti-CD45-BV421 (Biolegend \#103133),
anti-F4/80-APC780 (clone BM8, eBioscience \#47-4801-80), anti-CD206-APC (clone $\mathrm{C} 068 \mathrm{C} 2$, Biolegend \#141707), anti-Arg1-PECy7 (clone AlexF5, eBioscience \#25-3697-82), and anti-iNOS-PE (CXNPT, eBioscience \#12-5920-82). Panel 4 (for analysis of monocyte differentiation): anti-CD45-BV421 (Biolegend \#103133), anti-CD11b-PECy7 (clone M1/70, Biolegend \#101215), anti-Ly6C-APC (Biolegend \#128016), and anti-F4/80-APC780 (clone BM8, eBioscience \#47-4801-80). All antibodies were used at 1:100 dilution, apart from the anti-CD45 which was diluted 1:300. Between 300,000 and 500,000 cells were stained. Dead cells were stained with DAPI. For Arg1 and iNOS intracellular staining Intracellular Fixation and Permeabilization Buffer Set (eBioscience \#88-8824-00) was used by following manufacturer's indications, together with LIVE/DEAD Fixable Blue Dead Cell Stain (Invitrogen \#L34962). The samples were run on LSRFortessa cell analyzer (BD Biosciences) and data was analyzed by BD FACSDIVA Software (BD Bioscience) and Flow Jo (Tree Star Inc.) software.

Gel contraction assay. To assess fibroblast activation toward a CAF phenotype, normal murine-derived breast fibroblasts ${ }^{57}$ were seeded in 24-well dishes at 20,000 cells/well in $100 \mu \mathrm{L}$ of 2:1 Rat tail collagen-I (Corning \#354249) and Matrigel (Corning \#356234), yielding a final collagen concentration of $4 \mathrm{mg} \mathrm{mL}^{-1}$ and a final Matrigel concentration of $2 \mathrm{mg} \mathrm{mL}^{-1}$. Conditioned media from a $48 \mathrm{~h}$ culture of 100,000 cancer cells $(500 \mu \mathrm{L}=1 / 4$ of the volume), was added and geldetachment from the well edge was followed.

Cytokine array. Rag1 ${ }^{-I-} F V B / n$ Xenograft-derived cell cultures were generated by a 10-day cultivation of liberase-digested tissue in basal conditions. Supernatant $(0.5 \mathrm{~mL})$ was taken 2 days after medium renewal and analyzed with human Proteome Profiler Array kit (R\&D Systems, ARY005B) following manufacturer's instructions.

Statistical analysis. GraphPad Prism version 7 (GraphPad Software Inc., San Diego, CA, USA) was used to perform statistical tests and create bar plots and graphs. Unless stated otherwise, a two-tailed unpaired Student's $t$-tests assuming equal variance were performed to compare averages. When the F-test to compare variances between the two groups was significant, the data were transformed $\left(y^{\prime}=\log y\right)$ prior to the $t$-test calculus. In the few cases where log transformation did not correct variances, a $t$-test for unequal variances was applied. For each experiment, at least three biological replicates were analyzed. In vitro analyses were repeated by at least two independent experiments. In vivo experiments were repeated at least twice for data presented in Fig. 1a, Fig. 3g, Fig. 5a-c, Fig. 6b, Fig. 6d, Supplementary Fig. 16a, and Fig. 17c. For each experiment, $p$-values $\left({ }^{*} p<0.05,{ }^{* *} p<0.01,{ }^{* * *} p<0.001\right), t$-values $(t)$ and degrees of freedom (df) are indicated in figure legends, as well as the specification whether the log transformation or the $t$-test for unequal variances was performed. Moreover, where indicated, standard error of the mean (s.e.m.) is represented by the error bars. Survival curves were estimated using the Kaplan-Meier product-limit method and compared using a log-rank test (Mantel-Cox).

Reporting summary. Further information on experimental design is available in the Nature Research Reporting Summary linked to this article.

\section{Data availability}

The authors declare that data supporting the findings of this study are available within the paper and its supplementary information files.

Received: 7 June 2018 Accepted: 30 January 2019 Published online: 22 February 2019

\section{References}

1. Wheaton, W. W. et al. Metformin inhibits mitochondrial complex I of cancer cells to reduce tumorigenesis. eLife 3, e02242 (2014).

2. Gong, J. et al. The expanding role of metformin in cancer: an update on antitumor mechanisms and clinical development. Target. Oncol. 11, 447-467 (2016).

3. Chan, A. T. Metformin for cancer prevention: a reason for optimism. Lancet Oncol. 17, 407-409 (2016).

4. Koppenol, W. H., Bounds, P. L. \& Dang, C. V. Otto Warburg's contributions to current concepts of cancer metabolism. Nat. Rev. Cancer 11, 325-337 (2011).

5. Ju, Y. S. et al. Origins and functional consequences of somatic mitochondrial DNA mutations in human cancer. eLife 3, e02935 (2014).

6. Iommarini, L., Calvaruso, M. A., Kurelac, I., Gasparre, G. \& Porcelli, A. M. Complex I impairment in mitochondrial diseases and cancer: parallel roads leading to different outcomes. Int. J. Biochem. Cell. Biol. 45, 47-63 (2013). 
7. Ohta, S. Contribution of somatic mutations in the mitochondrial genome to the development of cancer and tolerance against anticancer drugs. Oncogene 25, 4768-4776 (2006).

8. Pereira, L., Soares, P., Maximo, V. \& Samuels, D. C. Somatic mitochondrial DNA mutations in cancer escape purifying selection and high pathogenicity mutations lead to the oncocytic phenotype: pathogenicity analysis of reported somatic mtDNA mutations in tumors. BMC Cancer 12, 53 (2012).

9. Zimmermann, F. A. et al. Respiratory chain complex I is a mitochondrial tumor suppressor of oncocytic tumors. Front. Biosci. Elite Ed. 3, 315-325 (2011).

10. Porcelli, A. M. et al. The genetic and metabolic signature of oncocytic transformation implicates HIF1alpha destabilization. Hum. Mol. Genet. 19, 1019-1032 (2010).

11. Gasparre, G., Porcelli, A. M., Lenaz, G. \& Romeo, G. Relevance of mitochondrial genetics and metabolism in cancer development. Cold Spring Harb. Perspect. Biol. 5, a011411 (2013).

12. Joshi, S. et al. The genomic landscape of renal oncocytoma identifies a metabolic barrier to tumorigenesis. Cell Rep. 13, 1895-1908 (2015).

13. Guo, J. Y. et al. Autophagy suppresses progression of K-ras-induced lung tumors to oncocytomas and maintains lipid homeostasis. Genes Dev. 27, 1447-1461 (2013).

14. Girolimetti, G. et al. Platinum-induced mitochondrial DNA mutations confer lower sensitivity to paclitaxel by impairing tubulin cytoskeletal organization. Hum. Mol. Genet. 26, 2961-2974 (2017).

15. Guerra, F. et al. Mitochondrial DNA mutation in serous ovarian cancer: implications for mitochondria-coded genes in chemoresistance. J. Clin. Oncol. 30, e373-e378 (2012).

16. Mullen, A. R. et al. Reductive carboxylation supports growth in tumour cells with defective mitochondria. Nature 481, 385-388 (2011)

17. Fendt, S.-M. et al. Metformin decreases glucose oxidation and increases the dependency of prostate cancer cells on reductive glutamine metabolism. Cancer Res. 73, 4429-4438 (2013).

18. Griss, T. et al. Metformin antagonizes cancer cell proliferation by suppressing mitochondrial-dependent biosynthesis. PLoS Biol. 13, e1002309 (2015).

19. Gasparre, G. et al. A mutation threshold distinguishes the antitumorigenic effects of the mitochondrial gene MTND1, an oncojanus function. Cancer Res. 71, 6220-6229 (2011).

20. Calabrese, C. et al. Respiratory complex I is essential to induce a Warburg profile in mitochondria-defective tumor cells. Cancer Metab. 1, 11 (2013).

21. Iommarini, L. et al. Different mtDNA mutations modify tumor progression in dependence of the degree of respiratory complex I impairment. Hum. Mol. Genet. 23, 1453-1466 (2014).

22. Grifoni, D. et al. The human protein Hugl-1 substitutes for Drosophila lethal giant larvae tumour suppressor function in vivo. Oncogene 23, 8688-8694 (2004).

23. Khan, S. J. et al. Epithelial neoplasia in Drosophila entails switch to primitive cell states. Proc. Natl Acad. Sci. USA 110, E2163-E2172 (2013).

24. Tal, R. et al. Activation of C-transactivation domain is essential for optima HIF-1 $\alpha$-mediated transcriptional and angiogenic effects. Microvasc. Res. 76, 1-6 (2008)

25. Kaelin, W. G. J. \& Ratcliffe, P. J. Oxygen sensing by metazoans: the central role of the HIF hydroxylase pathway. Mol. Cell 30, 393-402 (2008).

26. Iommarini, L., Porcelli, A. M., Gasparre, G. \& Kurelac, I. Non-canonical mechanisms regulating hypoxia-inducible factor 1 alpha in cancer. Front. Oncol. 7, 286 (2017).

27. Du, J. et al. PI3K and ERK-induced Racl activation mediates hypoxia-induced HIF-1a expression in MCF-7 breast cancer cells. PLoS ONE 6, e25213 (2011).

28. Milkiewicz, M., Pugh, C. W. \& Egginton, S. Inhibition of endogenous HIF inactivation induces angiogenesis in ischaemic skeletal muscles of mice. J. Physiol. 560, 21-26 (2004)

29. MacKenzie, E. D. et al. Cell-permeating alpha-ketoglutarate derivatives alleviate pseudohypoxia in succinate dehydrogenase-deficient cells. Mol. Cell. Biol. 27, 3282-3289 (2007)

30. Tennant, D. A. et al. Reactivating HIF prolyl hydroxylases under hypoxia results in metabolic catastrophe and cell death. Oncogene 28, 4009-4021 (2009).

31. Hagen, T., Taylor, C. T., Lam, F. \& Moncada, S. Redistribution of intracellular oxygen in hypoxia by nitric oxide: effect on HIFla. Science 302, 1975-1978 (2003)

32. De Palma, M., Biziato, D. \& Petrova, T. V. Microenvironmental regulation of tumour angiogenesis. Nat. Rev. Cancer 17, 457-474 (2017).

33. Castro, B. A. et al. Macrophage migration inhibitory factor downregulation: a novel mechanism of resistance to anti-angiogenic therapy. Oncogene 36, 3749-3759 (2017).

34. Comito, G. et al. Cancer-associated fibroblasts and M2-polarized macrophages synergize during prostate carcinoma progression. Oncogene 33, 2423-2431 (2014).

35. Jetten, $\mathrm{N}$. et al. Anti-inflammatory M2, but not pro-inflammatory M1 macrophages promote angiogenesis in vivo. Angiogenesis 17, 109-118 (2014).

36. Rolny, C. et al. HRG inhibits tumor growth and metastasis by inducing macrophage polarization and vessel normalization through downregulation of PlGF. Cancer Cell. 19, 31-44 (2011).
37. Akatsuka, A., Kojima, N., Okamura, M., Dan, S. \& Yamori, T. A novel thiophene-3-carboxamide analog of annonaceous acetogenin exhibits antitumor activity via inhibition of mitochondrial complex I. Pharmacol. Res. Perspect. 4, e00246 (2016).

38. Schockel, L. et al. Targeting mitochondrial complex I using BAY 87-2243 reduces melanoma tumor growth. Cancer Metab. 3, 11 (2015).

39. Higurashi, T. et al. Metformin for chemoprevention of metachronous colorectal adenoma or polyps in post-polypectomy patients without diabetes: a multicentre double-blind, placebo-controlled, randomised phase 3 trial. Lancet Oncol. 17, 475-483 (2016).

40. Kordes, S. et al. Metformin in patients with advanced pancreatic cancer: a double-blind, randomised, placebo-controlled phase 2 trial. Lancet Oncol. 16 839-847 (2015).

41. Bonanni, B. et al. Dual effect of metformin on breast cancer proliferation in a randomized presurgical trial. J. Clin. Oncol. 30, 2593-2600 (2012).

42. Coyle, C., Cafferty, F. H., Vale, C. \& Langley, R. E. Metformin as an adjuvant treatment for cancer: a systematic review and meta-analysis. Ann. Oncol. 27, 2184-2195 (2016)

43. Chak, A. et al. Metformin does not reduce markers of cell proliferation in esophageal tissues of patients with Barrett's esophagus. Clin. Gastroenterol. Hepatol. 13, 665-672 (2015). e1-4.

44. Hirata, E. \& Sahai, E. Tumor microenvironment and differential responses to therapy. Cold Spring Harb. Perspect. Med. 7, a026781 (2017).

45. Chen, P. \& Bonaldo, P. Role of macrophage polarization in tumor angiogenesis and vessel normalization: implications for new anticancer therapies. Int. Rev. Cell. Mol. Biol. 301, 1-35 (2013).

46. Leone, G., Abla, H., Gasparre, G., Porcelli, A. M. \& Iommarini, L. The oncojanus paradigm of respiratory complex I. Genes 9, 243 (2018).

47. Crane, M. J. et al. The monocyte to macrophage transition in the murine sterile wound. PLOS ONE 9, e86660 (2014).

48. Greaves, M. \& Maley, C. C. Clonal evolution in cancer. Nature 481, 306-313 (2012).

49. Trounce, I. A., Kim, Y. L., Jun, A. S. \& Wallace, D. C. Assessment of mitochondrial oxidative phosphorylation in patient muscle biopsies, lymphoblasts, and transmitochondrial cell lines. Methods Enzymol. 264, 484-509 (1996).

50. Moreno-Loshuertos, R. et al. Differences in reactive oxygen species production explain the phenotypes associated with common mouse mitochondrial DNA variants. Nat. Genet. 38, 1261-1268 (2006).

51. Bustin, S. A. et al. The MIQE guidelines: minimum information for publication of quantitative real-time PCR experiments. Clin. Chem. 55, 611-622 (2009).

52. Rozen, S. \& Skaletsky, H. Primer3 on the WWW for general users and for biologist programmers. Methods Mol. Biol. Clifton NJ 132, 365-386 (2000).

53. Zuker, M. Mfold web server for nucleic acid folding and hybridization prediction. Nucleic Acids Res. 31, 3406-3415 (2003).

54. Yuan, J. S., Reed, A., Chen, F. \& Stewart, C. N. Statistical analysis of real-time PCR data. BMC Bioinform. 7, 85 (2006).

55. Grifoni, D., Sollazzo, M., Fontana, E., Froldi, F. \& Pession, A. Multiple strategies of oxygen supply in Drosophila malignancies identify tracheogenesis as a novel cancer hallmark. Sci. Rep. 5, 9061 (2015).

56. Wculek, S. K. \& Malanchi, I. Neutrophils support lung colonization of metastasis-initiating breast cancer cells. Nature 528, 413-417 (2015).

57. Del Pozo Martin, Y. et al. Mesenchymal cancer cell-stroma crosstalk promotes niche activation, epithelial reversion, and metastatic colonization. Cell Rep. 13 2456-2469 (2015)

\section{Acknowledgements}

This work was supported by EU H2020 Marie Curie project TRANSMIT GA 722605 and EU FP7 Marie Curie ITN-317433 MEET to A.M.P. and G. Gasparre; by Associazione Italiana Ricerca sul Cancro (AIRC) grant JANEUTICS-IG14242 and Italian Ministry of Health grant DISCO TRIP GR-2013-02356666 to G. Gasparre. G. Girolimetti was supported by AIRC triennal fellowhip "Livia Perotti" and M.D.L. by triennial AIRC fellowship "Bruna Martelli". This work was also supported by The Francis Crick Institute which receives its core funding from Cancer Research UK (FC001112), the UK Medical Research Council (FC001112), and the Wellcome Trust (FC001112). We thank the Biomedical Research Facility, Experimental Histopathology and Flow cytometry units from The Francis Crick Institute (UK). We are also grateful to Sepideh AminzadehGohari, Felix Locker, Simone Di Giacomo, Patty Wai and Claudia Calabrese for technical help. We finally thank Christine M. Betts for English language editing and Pasquale Chieco for the help with statistical analyses.

\section{Author contributions}

I.K. and L.B.A. created the NDUFS3 ${ }^{-1-}$ models and participated in the generation of inducible NDUFS 3 knockout and TM-HIF-1 $\alpha$ models. I.K. evaluated HIF-1 $\alpha$ protein levels and HIF1-responsive gene expression. L.I., G.L. and R.V. performed biochemical experiments. I.K. and R.V. performed in vivo studies and histology analyses. M.D.L. participated in creation of the inducible NDUFS3 knockout model. G. Girolimetti, N.U.G., V.L.B., R.G.F., S.V., L.O. and M.B. provided technical help for in vivo studies 
M.C. performed electron microscopy analyses. M.R. is the pathologist who evaluated all histology data. I.K. performed flow cytometry experiments, for which L.O. and I.M. helped with data analysis. L.G. and A.C. performed vector transductions. M.V. created the vector containing TM-HIF-1a. M.S., S.F. and D.G. performed fly experiments. A.M.P. and G. Gasparre conceived the study. I.K., L.I., A.M.P. and G. Gasparre wrote the manuscript. L.O., I.M., B.K., R.V. and S.F. critically revised the manuscript.

\section{Additional information}

Supplementary Information accompanies this paper at https://doi.org/10.1038/s41467019-08839-1.

Competing interests: The authors declare no competing interests.

Reprints and permission information is available online at http://npg.nature.com/ reprintsandpermissions/

Journal peer review information: Nature Communications thanks Giulio Draetta, Leila Akkari and the other anonymous reviewer(s) for their contribution to the peer review of this work. Peer reviewer reports are available.
Publisher's note: Springer Nature remains neutral with regard to jurisdictional claims in published maps and institutional affiliations.

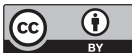

Open Access This article is licensed under a Creative Commons Attribution 4.0 International License, which permits use, sharing, adaptation, distribution and reproduction in any medium or format, as long as you give appropriate credit to the original author(s) and the source, provide a link to the Creative Commons license, and indicate if changes were made. The images or other third party material in this article are included in the article's Creative Commons license, unless indicated otherwise in a credit line to the material. If material is not included in the article's Creative Commons license and your intended use is not permitted by statutory regulation or exceeds the permitted use, you will need to obtain permission directly from the copyright holder. To view a copy of this license, visit http://creativecommons.org/ licenses/by/4.0/.

(C) The Author(s) 2019 